ARTICLE

\title{
Thiazoline-related innate fear stimuli orchestrate hypothermia and anti-hypoxia via sensory TRPA1 activation
}

Tomohiko Matsuo ${ }^{1}$, Tomoko Isosaka1, Yuichiro Hayashi ${ }^{1}$, Lijun Tang ${ }^{1}$, Akihiro Doi id ${ }^{1}$, Aiko Yasuda , Mikio Hayashi (1) ${ }^{2}$, Chia-Ying Lee ${ }^{3}$, Liqin $\mathrm{Cao}^{3}$, Natsumaro Kutsuna ${ }^{4,5}$, Sachihiro Matsunaga (1) ${ }^{6}$, Takeshi Matsuda7, Ikuko Yao7 , Mitsuyoshi Setou ${ }^{8}$, Dai Kanagawa1, Koichiro Higasa9, Masahito Ikawa (10 ${ }^{10,}$ Qinghua Liu (i) ${ }^{3,11 凶}$, Reiko Kobayakawa (i) ${ }^{1 凶} \&$ Ko Kobayakawa (i) ${ }^{1 凶}$

Thiazoline-related innate fear-eliciting compounds (tFOs) orchestrate hypothermia, hypometabolism, and anti-hypoxia, which enable survival in lethal hypoxic conditions. Here, we show that most of these effects are severely attenuated in transient receptor potential ankyrin 1 (Trpa1) knockout mice. TFO-induced hypothermia involves the Trpa1-mediated trigeminal/vagal pathways and non-Trpa1 olfactory pathway. TFOs activate Trpa1-positive sensory pathways projecting from trigeminal and vagal ganglia to the spinal trigeminal nucleus (Sp5) and nucleus of the solitary tract (NTS), and their artificial activation induces hypothermia. TFO presentation activates the NTS-Parabrachial nucleus pathway to induce hypothermia and hypometabolism; this activation was suppressed in Trpa1 knockout mice. TRPA1 activation is insufficient to trigger tFO-mediated anti-hypoxic effects; Sp5/NTS activation is also necessary. Accordingly, we find a novel molecule that enables mice to survive in a lethal hypoxic condition ten times longer than known tFOs. Combinations of appropriate tFOs and TRPA1 command intrinsic physiological responses relevant to survival fate.

\footnotetext{
${ }^{1}$ Department of Functional Neuroscience, Institute of Biomedical Science, Kansai Medical University, Osaka, Japan. ${ }^{2}$ Department of Cellular and Functional Biology, Institute of Biomedical Science, Kansai Medical University, Osaka, Japan. ${ }^{3}$ International Institute for Integrative Sleep Medicine (WPI-IIIS), University of Tsukuba, Tsukuba, Ibaraki, Japan. ${ }^{4}$ Department of Integrated Biosciences, Graduate School of Frontier Sciences, University of Tokyo, Chiba, Japan. ${ }^{5}$ LPixel Inc., Tokyo, Japan. ${ }^{6}$ Department of Applied Biological Science, Faculty of Science and Technology, Tokyo University of Science, Chiba, Japan. ${ }^{7}$ Department of Optical Imaging, Institute for Medical Photonics Research, PMPERC and IMIC, Hamamatsu University School of Medicine, Shizuoka, Japan. ${ }^{8}$ Department of Cellular and Molecular Anatomy and IMIC, Hamamatsu University School of Medicine, Shizuoka, Japan. ${ }^{9}$ Department of Genome Analysis, Institute of Biomedical Science, Kansai Medical University, Osaka, Japan. ${ }^{10}$ Research Institute for Microbial Diseases, Osaka University, Osaka, Japan. ${ }^{11}$ National Institute of Biological Sciences, Beijing, China. ${ }^{凶}$ email: linqinghua@nibs.ac.cn; kobayakr@hirakata.kmu.ac.jp; kobayakk@hirakata.kmu.ac.jp
} 
$\mathrm{n}$ life-threatening situations, organisms protect themselves using latent bioprotective capabilities. The diving reflex, induced by trigeminal activation by cold immersion in neardrowning, and the vagal reflex, induced by fear perception, cause considerable fluctuations in the homeostatic state in humans ${ }^{1,2}$. These reflexes have supposedly evolved as life-protective abilities; however, clinical applications utilizing these effects have yet to be established.

Innate fear is intimately connected to the preservation of life. However, the absence of its effective inducers prevents uncovering latent protective abilities. By optimizing the chemical structure of 2,4,5-trimethyl-3-thiazoline (TMT) ${ }^{3}$, a predatorderived compound and innate fear inducer in rodents, we developed artificial compounds, thiazoline-related fear-eliciting compounds (tFOs), e.g., 2-methyl-2-thiazoline (2MT), inducing potent innate fear response in mice 4 . We recently found that tFOs orchestrate robust hypothermia, anaerobic metabolism, and anti-hypoxic responses, which extended survival in lethal hypoxic conditions and decreased the severity of ischemia/ reperfusion models ${ }^{5}$. Thus, identifying the receptor gene responsible for these physiological responses induced by tFOs is important to evaluate whether the phenomena can be used for medical applications.

Previous studies have identified several candidate receptor genes and neural pathways in the perception of tFOs. The dorsal olfactory pathway and its odorant receptors regulate avoidance and fear-related behaviors induced by TMT and the alarm pheromone 2-sec-butyl-2-thiazoline (SBT) ${ }^{6-8}$. On the other hand, forward genetic screenings identified that freezing and avoidance behaviors induced by tFOs, such as $2 \mathrm{MT}$ and TMT, were regulated by the transient receptor potential ankyrin 1 (Trpa1) gene in trigeminal neurons ${ }^{9}$. Furthermore, fear-related behaviors were also suppressed in $\operatorname{Trpa1} 1^{-1-}$ mice in response to a natural product, e.g., snake-derived compounds. Thus, fear-related behaviors in response to tFOs are thought to be regulated by at least two different systems: (1) Trpal in the trigeminal neurons and (2) odorant receptors in the main olfactory system. However, the genes and neural pathways responsible for tFO-induced physiological effects are unknown. Here, we aimed to clarify the contribution of Trpal in regulating these effects.

TRPA1 was initially identified as a cold-activated ion channel ${ }^{10}$. TRPA1 is also activated by extrinsic stimuli, such as allyl isothiocyanate (AITC), a pungent component of mustard oil and wasabi, and formalin, a nociceptive stimulus ${ }^{11,12}$, as well as by intrinsic stimuli, such as 4-hydroxy-2-nonenal and $\mathrm{H}_{2} \mathrm{O}_{2}$ generated by inflammation ${ }^{13,14}$. Furthermore, Trpal is involved in inflammatory pain and hypersensitivity after inflammation ${ }^{15-17}$, in the perception of aberrant oxygen concentration, and in the regulation of respiratory responses to mild hypoxia ${ }^{18,19}$. Collectively, Trpa1 is considered an alarm sensor detecting multiple signals to transduce pain or danger information to the brain $18,20,21$. Extending these findings, we hypothesized that Trpa1 also has a crucial role in tFO-mediated latent physiological responses, which increase survival in lethal conditions. We found that tFOs are perceived by Trpal in the trigeminal and vagus nerves. This information is transmitted to the spinal trigeminal tract (Sp5) and the nucleus of the solitary tract (NTS) to regulate hypothermia, hypoxic metabolism, and survivability in lethal hypoxic conditions. Finally, by monitoring the activation of TRPA1 and Sp5/NTS, we identified a novel compound that could prolong survival in hypoxic conditions ten times longer than known tFOs. Taken together, our results indicate that Trpal not only functions as a danger sensor, but also commands the induction of physiological responses relevant to innate fear, and is even involved in the acquisition of viability in lethal hypoxic conditions.

\section{Results}

tFO induces hypothermia/anti-hypoxia via Trpa1. Avoidance and risk assessment behaviors relevant to innate fear induced by $2 \mathrm{MT}$ are regulated by Trpa1 (refs. ${ }^{4,9}$ ). $2 \mathrm{MT}$ also induces robust physiological responses such as hypothermia and bradycardia 5 . We examined whether these physiological responses are regulated by Trpa1 using Trpa $1^{-1-}$ mice. Whereas body temperature in the baseline condition was not significantly altered, 2MT-induced hypothermia was greatly suppressed in $\operatorname{Trpa1}^{-1-}$ mice (Fig. 1a, b). Most Trpa1-expressing cells co-express Trpv1 (ref. ${ }^{10}$ ). However, 2MT-induced hypothermia was not affected in $\operatorname{Trp} v 1^{-1-}$ mice (Fig. 1c). Hypothermia is observed not only because of tFO stimulation but also in response to restraint in a tight space, another type of innate fear stimulus ${ }^{5}$. If Trpal works as a peripheral sensor for $2 \mathrm{MT}$ to induce hypothermia, it is expected that hypothermia induced by other types of innate fear stimuli would not be affected in Trpa1 ${ }^{-1-}$ mice. As expected, restraint in a tight place induced hypothermia in Trpa1 ${ }^{-/}$mice (Fig. 1d). Similarly, the heart rate in the control condition was not altered, but quick and robust bradycardia following $2 \mathrm{MT}$ presentation was almost completely suppressed in Trpa1 ${ }^{-1-}$ mice (Fig. 1e). In contrast, reduction of heart rate induced by restraint in a tight space was observed in Trpa1 $1^{-1-}$ mice (Fig. 1f). Interestingly, hypothermia and bradycardia induced by restraint in a tight space tended to be greater in Trpa1 ${ }^{-1-}$ mice than those in control mice. Thus, it is suggested that the Trpal gene contributes to the initiation of innate fearrelevant suppression of body temperature/heart rate; Trpa1 may also have important roles in maintaining appropriate suppression levels to various stimuli.

Under normal conditions, mice cannot survive in a hypoxic environment, whereas it is reported that hydrogen sulfide $\left(\mathrm{H}_{2} \mathrm{~S}\right)$ prolongs the survival time in such environment ${ }^{22}$. High concentrations of $\mathrm{H}_{2} \mathrm{~S}$ lead to the death of individuals by inhibiting the activity of the electron transfer chain in the mitochondria ${ }^{23}$, which is essential for oxygen respiration. Low concentration of $\mathrm{H}_{2} \mathrm{~S}$ is thought to partially inhibit mitochondrial respiratory activity, thereby enhancing the ability to survive in a hypoxic environment ${ }^{22,24}$. On the other hand, 2MT has no inhibitory effects on the mitochondrial respiratory chain. Nevertheless, 2MTstimulation evokes hypoxic metabolism and hypoxia resistance ability $^{5}$. How does 2MT induce hypoxia resistance without inhibiting the mitochondrial respiratory chain? As we reported previously, oxygen consumption was suppressed by presentation of 2MT. Importantly, prior presentation of $2 \mathrm{MT}$ increased the survival time in lethal $4 \%$ oxygen conditions ${ }^{5}$. Then, we asked if these impacts of $2 \mathrm{MT}$ presentation on oxygen metabolism are also controlled by Trpa1 gene. In Trpa1 ${ }^{-1-}$ mice, suppression of oxygen consumption induced by $2 \mathrm{MT}$ presentation was completely suppressed (Fig. 2a). Further, survival time in $4 \%$ oxygen condition was significantly shortened in $2 \mathrm{MT}-\operatorname{Trpa1} 1^{-1-}$ mice (Fig. 2b). These results suggest that $2 \mathrm{MT}$ causes hypometabolism to fulfill antihypoxic effects through Trpa1. These results also raise the possibility Trpa1 agonists other than tFOs might induce antihypoxic effects. To test this possibility, we analyzed the effects of previously reported Trpal agonists: trans- $\Delta^{9}$-tetrahydrocannabinol, a component of cannabis; AITC; and acetaminophen, an analgesic antipyretic $^{11,25,26}$. Survival time under hypoxic conditions was prolonged by these ligands (Fig. 2c), and acetaminophen induced the strongest effect, which was absent in $\operatorname{Trpa1}^{-1-}$ mice (Fig. 2d). Collectively, TRPA1 activation by diverse ligands orchestrate hypothermia, hypometabolism, and anti-hypoxic effects.

Multiple sensory pathways are involved in tFO-induced hypothermia. Both the main olfactory neurons and the trigeminal neurons regulate fearful behaviors evoked by 

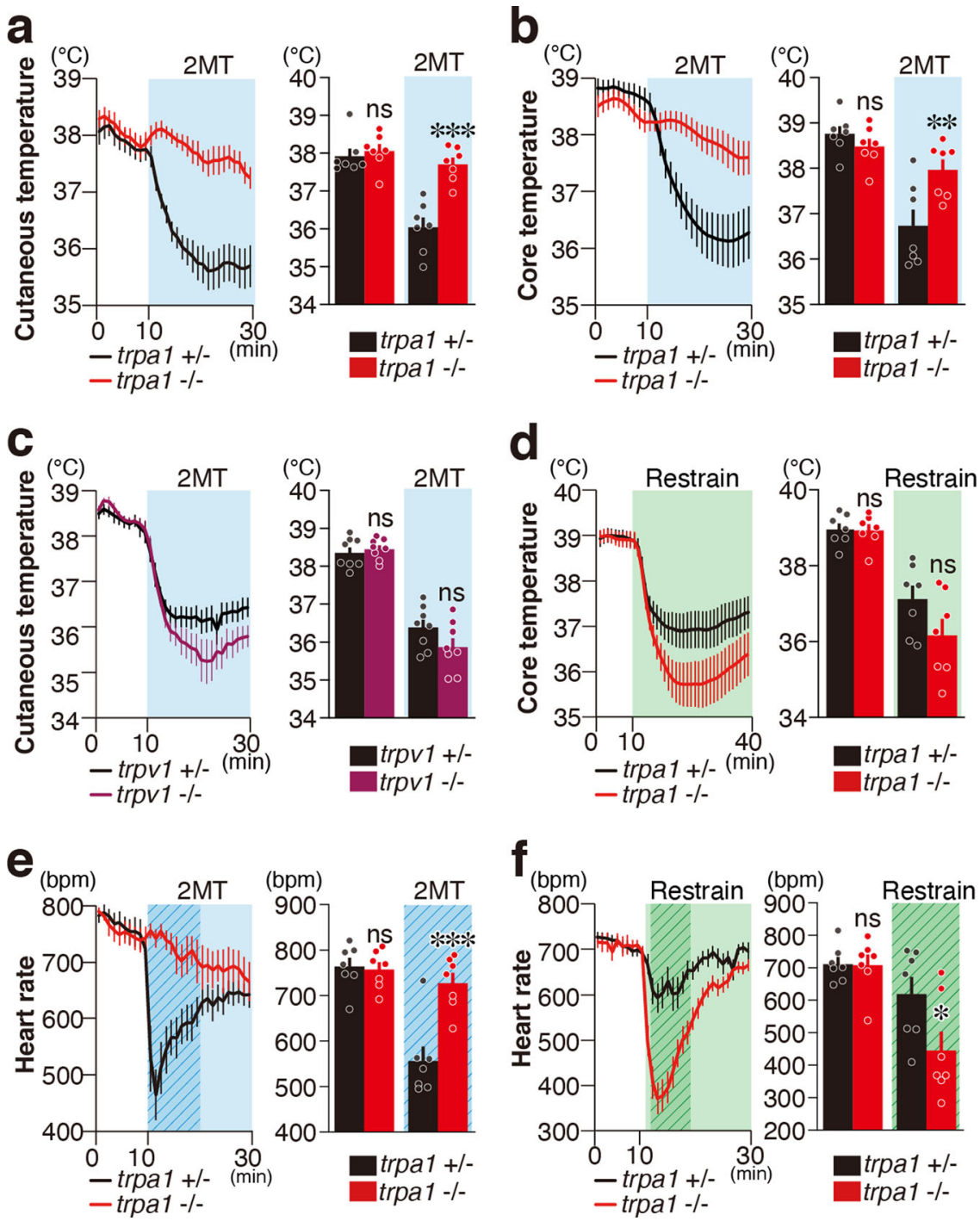

Fig. 1 Trpa1 mediates tFO-evoked hypothermia and bradycardia. a, b Cutaneous temperature (a) and core body temperature (b) temporal analysis in Trpa1 $^{-1-}$ (red) and control (black) mice in response to presentation of 2-methyl-2-thiazoline (2MT) ( $\mathbf{a}, n=7$ for each genotype; $\mathbf{b}, n=7$ for each genotype). Mean cutaneous/core temperature in $10 \mathrm{~min}$ of baseline session and $20 \mathrm{~min}$ of $2 \mathrm{MT}$ presentation are also shown (a, $p=0.8708$ for baseline session and $p<0.0001$ for during 2 MT presentation; $\mathbf{b}, p=0.6638$ for baseline session and $p=0.0026$ for 2 MT session). c Cutaneous temperature temporal analysis in Trpv1 ${ }^{-1-}$ (purple) and control (black) mice in response to $2 \mathrm{MT}$ presentation ( $n=8$ for each genotype). Mean cutaneous temperature in $10 \mathrm{~min}$ of baseline session and $20 \mathrm{~min}$ of 2 MT presentation are also shown ( $p=0.9121$ for baseline session and $p=0.0935$ for 2 MT session). $\mathbf{d}$ Core body temperature temporal analysis in Trpa1-/- (red) and control (black) mice in response to the restrained condition ( $n=7$ for each genotype). Mean core temperature during baseline session (1-10 $\mathrm{min}, p=0.9981)$ and during restrained condition (12-40 min, $p=0.0631)$ are also shown. e, $\mathbf{f}$ Temporal analysis of heart rates in Trpa1-/- (red) and control (black) mice in response to $2 \mathrm{MT}$ presentation (e, $n=7$ for each) and in restrained condition (f, $n=7$ for each). Mean heart rate in response to $2 \mathrm{MT}$ presentation $[\mathbf{e}, p=0.9757$ for baseline session (1-10 min) and $p<0.0001$ for $2 \mathrm{MT}$ session (11-20 min, marked by shaded duration in the left figure)] and the restrained condition [f, $p=0.9987$ for baseline (1-10 min) and $p=0.01084$ for restrained condition (12-20 min, marked by shaded areas in the left figure)] are also shown. Data are shown as mean \pm SEM. Two-way ANOVA followed by Sidak's multiple comparison test was used to assess significance; ${ }^{\star} p<0.05 ;{ }^{* \star} p<0.01 ;{ }^{* \star \star} p<0.001$; ns $p>0.05$.

predator-derived compounds and $\mathrm{FFOs}^{6-8}$. However, the contribution of these neurons in regulating tFO-induced physiological responses is unclear. In olfactory bulbectomized $(\mathrm{OBx})$ mice, cutaneous temperature reduction in response to $2 \mathrm{MT}$ exposure was suppressed compared to controls (Fig. 3a and Supplementary Fig. 1). The surgical removal of the olfactory bulbs (OBs) might injure parts of the trigeminal nerve innervating into the nasal cavity. To exclude this possibility, we analyzed the $\Delta \mathrm{D}$ mutant mice, in which the dorsal zone olfactory sensory neurons (OSNs) that regulate avoidance behaviors to predator odorants are genetically depleted ${ }^{6}$. We observed suppressed cutaneous temperature reduction in response to 2MT presentation (Fig. 3b).
The cyclic nucleotide-gated olfactory (Cnga2) channel is critical for OSNs to generate odor-induced action potentials ${ }^{27}$. We also analyzed the $\Delta \mathrm{D}$ (cng) mice, in which Cnga2 channel is deleted in the dorsal $\mathrm{OSN}^{7}$. Cutaneous temperature reduction was suppressed in these animals (Supplementary Fig. 2). Next, we analyzed the contribution of the trigeminal system in regulating cutaneous temperature. Mice receiving bilateral trigeminal ganglion (TG) electrocauterization died several days after surgery. In mice that received unilateral TG electrocauterization (ulTGx), we observed suppressed reduction of cutaneous temperature in response to 2MT, although contralateral TG and OBs are intact in these animals (Fig. 3c). Mice that received bilateral vagal nerve 

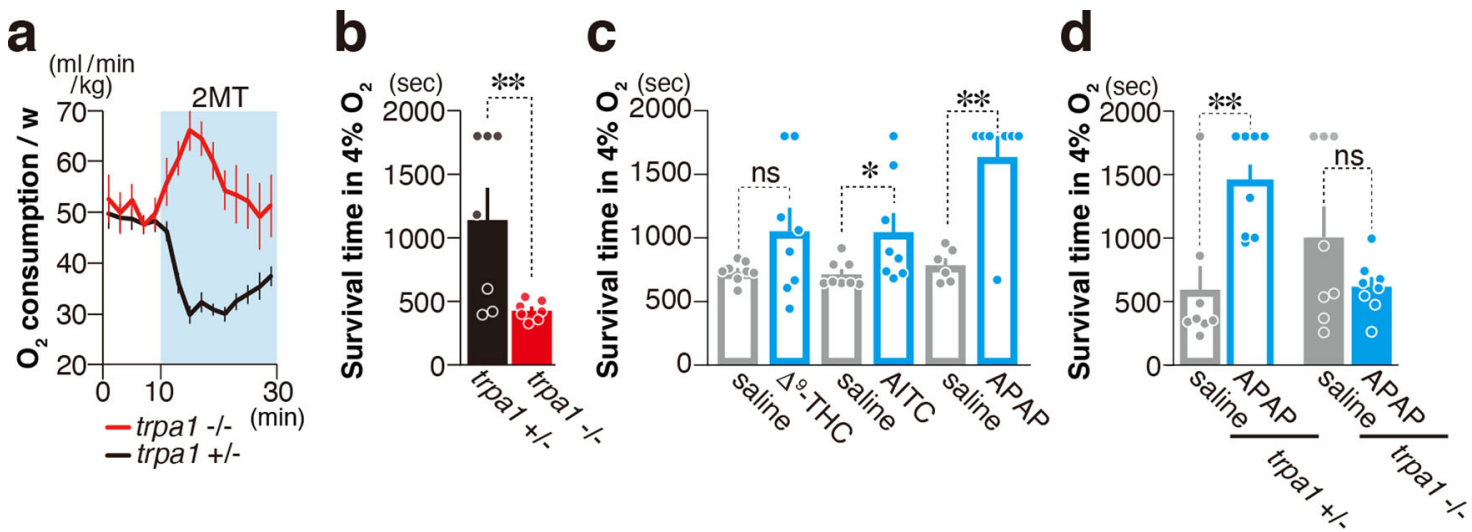

Fig. 2 Trpa1 mediates tFO-evoked anti-hypoxia. a Temporal analysis of oxygen consumption in Trpa1-/- (red) and control (black) mice in response to

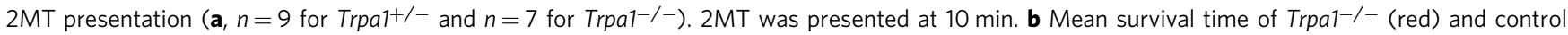
(black) mice in $4 \%$ oxygen with 10 min of prior 2 MT presentation $(n=7$ for each genotype, $p=0.0122)$. c Mean survival time of wildtype mice in $4 \%$ oxygen with (blue) and without (gray) intraperitoneal (IP) administration of the indicated compounds $\left[\Delta^{9}\right.$-tetrahydrocannabinol (THC); $n=8$ for each, $p=0.0586$ : isothiocyanate (AITC) $n=8$ each, $p=0.0258$ : acetaminophen (APAP); $n=6$ for saline and $n=7$ for APAP, $p=0.003$ ]. $\mathbf{d}$ Mean survival time of Trpa1 ${ }^{-/-}$(closed bars; $n=8$ each) and control (open bars; $n=8$ each) mice in $4 \%$ oxygen with (blue) and without (gray) IP administration of APAP; $p=0.0066$ for $\operatorname{Trpa}^{+/-}$and $p=0.2263$ for Trpa1 $^{-/}{ }^{-}$. Data are shown as mean \pm SEM. Log-rank test was used to assess statistical significance; ${ }^{\star} p<0.05$, ${ }^{\star \star} p<0.01,{ }^{\star \star \star} p<0.001$.

transection at the cervical level also died immediately after surgery. In contrast to ulTGx, reduction of cutaneous temperature in response to $2 \mathrm{MT}$ presentation was not affected in mice receiving unilateral transection (Supplementary Fig. 3a). Then, we ablated the bilateral subdiaphragmatic vagus nerves (VNSs), and again observed suppression of 2MT-induced cutaneous temperature reduction (Supplementary Fig. 3b). Taken together, these results indicate that the main olfactory, trigeminal, and vagus systems are involved in $\mathrm{tFO}$-induced hypothermia.

Trpa1 is expressed not only in trigeminal and vagus nerves, but also in the olfactory epithelium ${ }^{11,28,29}$. Therefore, we subsequently addressed which of these systems are involved in the regulation of Trpal-dependent tFO-induced hypothermia. Reduction of cutaneous temperature in response to $2 \mathrm{MT}$ was not affected in conditional knockout mice in which Trpa1 was removed in OSNs by using olfactory marker protein (OMP)-Cre mice $^{30}$ (Fig. 3d). However, this temperature reduction was suppressed in mutant mice in which Trpal was removed in the peripheral sensory neurons other than OSNs using Advillin-Cre mice $^{31}$ (Fig. 3e). In trigeminal neurons, most Trpa1 ${ }^{+}$neurons coexpress Trpv1 (ref. ${ }^{11}$ ). The administration of resiniferatoxin (RTX), an ultrapotent TRPV1 agonist, leads to cytotoxic calcium overload and cell death of $\operatorname{Trpv1}^{+}$neurons ${ }^{32,33}$. RTX administration in the TG suppressed 2MT-induced hypothermia (Fig. 3f). These results indicate that multiple sensory inputs, including the olfactory, trigeminal, and vagus nerves, are involved in tFOinduced hypothermia.

Vagal and trigeminal afferent projections of $\operatorname{Trpa1}^{+}$neurons. Hypothermia is regulated by the median preoptic nucleus (MnPO) and ventromedial preoptic area (VMPO) neurons in the hypothalamus ${ }^{34-37}$; hence, $2 \mathrm{MT}$-induced hypothermia may similarly be regulated by these brain regions. To test this possibility, we analyzed the c-fos mRNA expression in control and Trpa1 ${ }^{-1-}$ mice in response to 2MT presentation (Supplementary Fig. $4 \mathrm{a}-\mathrm{c})$. 2MT stimulation upregulated $c$-fos mRNA expression in the VMPO. However, this increased expression was unaffected in Trpa1 $1^{-1-}$ mice, suggesting that $2 \mathrm{MT}$-induced hypothermia mediated by Trpal is regulated by brain regions other than the $\mathrm{MnPO}$ and VMPO in the hypothalamus. Recently, we showed that 2MT activates the neuronal pathway from the NTS in the brainstem to the parabrachial nucleus (PBN) in the midbrain, and that artificial activation of this pathway induced hypothermia ${ }^{5}$. Thus, in this study, we considered that 2MT-induced activation of the NTS-PBN pathway may be regulated by Trpa1 (Supplementary Fig. $4 \mathrm{~d}-\mathrm{g}$ ). We found that $2 \mathrm{MT}$-induced $c$-fos mRNA expression in the PBN and NTS was almost completely abolished in Trpa1 $1^{-1-}$ mice, indicating that $2 \mathrm{MT}$-induced activation of the NTS-PBN pathway is mediated by Trpa1. These results suggest that 2MT-induced hypothermia mediated by Trpa1 is regulated by the brainstem-midbrain pathway rather than the known hypothalamic thermoregulatory center. Thus, we aimed to elucidate the signaling pathway of Trpa1 ${ }^{+}$neurons to the brainstem.

2MT presentation induced expression of $c$-fos, a neural activity marker, in both Sp5 and $\mathrm{NTS}^{5}$, areas which receive axonal projections mainly from the trigeminal ${ }^{38,39}$ and vagus nerves ${ }^{40-42}$. Trpal is expressed in a subset of trigeminal and vagus nerve neurons ${ }^{11,28}$, whose projection sites are not fully characterized. To address this question, we generated knock-in mice, in which the coding sequence of the Trpal gene was replaced with that of the Cre gene. To visualize Trpal-expressing cells, we crossed Trpa1-Cre mice with a reporter mouse strain expressing enhanced yellow fluorescent protein (EYFP) in a Credependent manner (Fig. 4a). The cell bodies of EYFP ${ }^{+}$neurons were observed in both the trigeminal and vagus ganglia (TG and VG) by whole-mount imaging (Fig. $4 b_{1}, b_{2}, c_{1}, c_{2}$ ). Histochemical analysis of TG/VG sections confirmed EYFP expression in smallsized cells as reported previously ${ }^{10,28}$ (Fig. $4 b_{3}, c_{3}$ ). Next, we analyzed the axonal projection of Trpa1 ${ }^{+}$neurons in the Sp5. EYFP $^{+}$fibers were found in the dorsal area of the spinal trigeminal nucleus caudalis $(\mathrm{Sp} 5 \mathrm{C})$ and in the ventral area of the spinal trigeminal nucleus interpolaris (Sp5I) (Fig. $\left.4 \mathrm{~d}_{1}-\mathrm{d}_{3}\right) ; c-f o s$ expression was observed in these areas after $\mathrm{tFO}$ stimulation (Fig. $4 \mathrm{e}_{1}-\mathrm{e}_{3}, \mathrm{f}_{1}-\mathrm{f}_{3}$ ). Furthermore, immunohistochemical analysis of phosphorylated ERK (pERK) ${ }^{43}$, another neural activity marker, showed EYFP ${ }^{+}$fibers surrounding the $\mathrm{pERK}^{+}$cells in tFOstimulated reporter mice (Fig. 4g-l). Next, we analyzed the area postrema (AP) and the NTS. The AP and the caudal NTS receive axonal inputs from the VNS, while the rostral NTS from the taste/ orofacial tactile nerves ${ }^{40,41}$. We observed $\mathrm{EYFP}^{+}$fibers in the caudal but not in the rostral NTS in reporter mice (Supplementary Fig. 5); $\mathrm{EYFP}^{+}$signal was especially strong in the dorsal area 

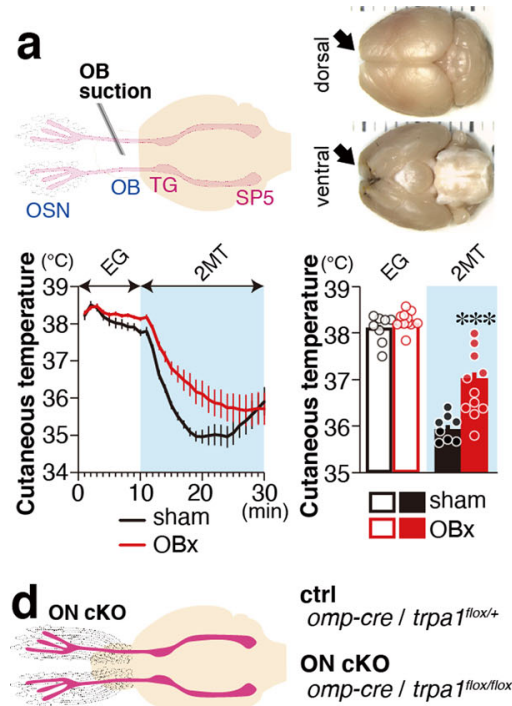

ON cKO

omp-cre / trpa1tioxilox
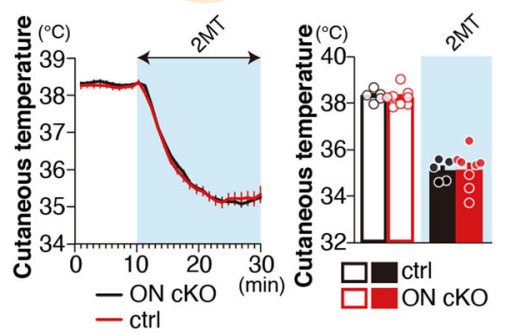
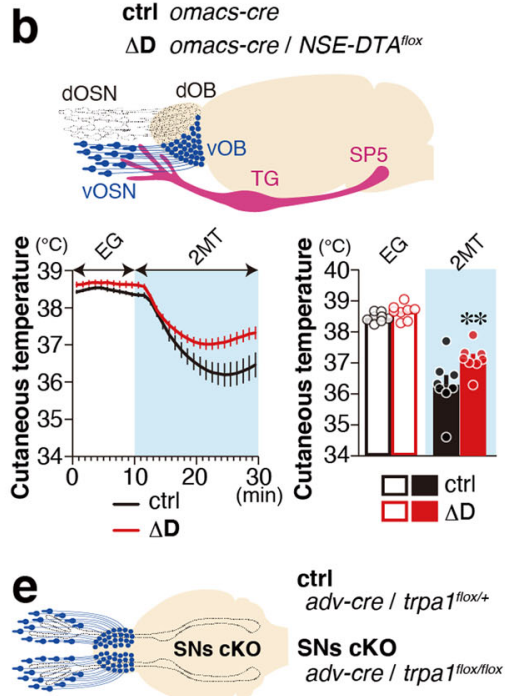

adv-cre /

adv-cre / trpa1 $1^{\text {floxflox }}$
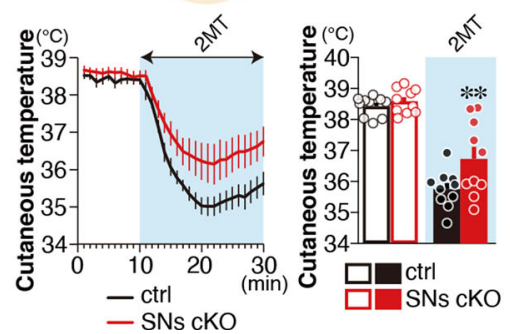
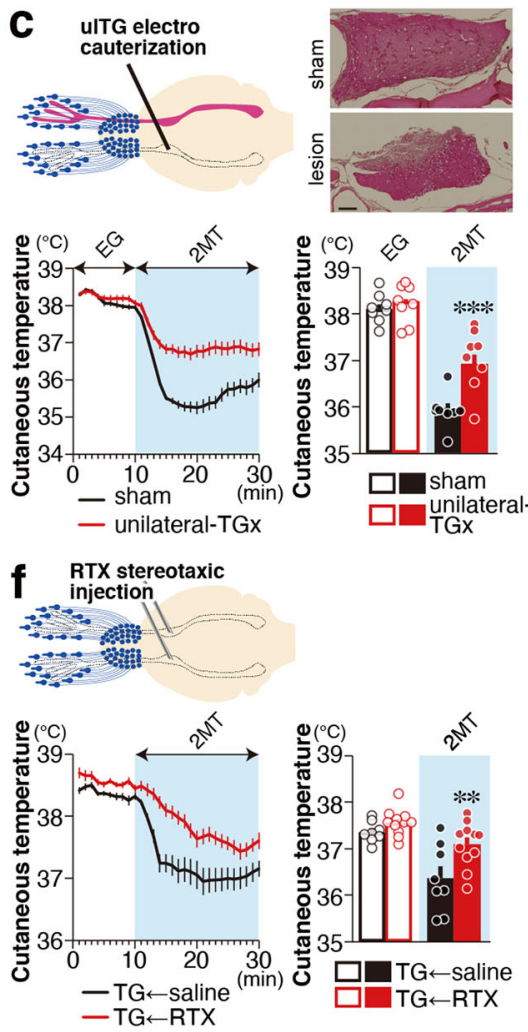

Fig. 3 Multiple sensory pathways are involved in tFO-induced hypothermia. a Temporal and mean cutaneous temperature of olfactory bulbectomized $(\mathrm{OBx} ; n=12)$ and sham-operated $(n=8)$ animals in response to eugenol (EG) and $2 \mathrm{MT}$ presentation are shown; $p=0.6302$ for eugenol presentation (1-10 min) and $p<0.0001$ for 2MT presentation (11-20 min). b Temporal and mean cutaneous temperature of $\Delta \mathrm{D}$ (Omacs-Cre; NSE-DTAflox) and control mice ( $n=8$ each) in response to EG and $2 \mathrm{MT}$ presentation are shown; $p=0.722$ for EG presentation ( $1-10$ min) and $p=0.0052$ for $2 \mathrm{MT}$ presentation (21-30 min). c Temporal and mean cutaneous temperature of unilateral trigeminal ganglion (TG)-lesioned and sham-operated animals ( $n=8$ each) in response to EG and 2MT presentation are shown; $p=0.8921$ for EG presentation (1-10 min) and $p<0.0001$ for $2 \mathrm{MT}$ presentation (11-20 min). d Temporal and mean cutaneous temperature of olfactory neuron (ON)-specific Trpa1 knockout (Omp-Cre; Trpafflox/flox) and control mice ( $n=5$ for control and $n=8$ for knockout) in response to 2 MT presentation are shown; $p=0.9757$ for baseline condition (1-10 min) and $p=0.9943$ for $2 \mathrm{MT}$ presentation ( $21-30$ min). e Temporal and mean cutaneous temperature of sensory neuron (SN)-specific Trpa1 knockout (Adv-Cre; Trpafflox/flox) and control mice ( $n=10$ each) are shown; $p=0.9775$ for baseline condition (1-10 min) and $p=0.0249$ for 2 MT presentation (21-30 min). f Temporal and mean cutaneous temperature of intra-trigeminal ganglion injection of saline and RTX ( $n=8$ for saline and $n=11$ for RTX) in response to $2 \mathrm{MT}$ presentation are shown; $p=0.5719$ for baseline condition (1-10 min) and $p=0.0034$ for 2MT presentation (11-20 min). Data are shown as mean \pm SEM. Two-way ANOVA followed by Sidak's multiple comparison test; ${ }^{\star \star} p<0.01 ;{ }^{\star \star \star} p<0.001$. Scale bars, $100 \mu \mathrm{m}$.

of the caudal NTS. Relatively weak $\mathrm{EYFP}^{+}$signals were observed in the AP (Fig. $4 \mathrm{~d}_{4}$ ). Meanwhile, $c$-fos expression was observed in the caudal NTS and AP region, where $\mathrm{EYFP}^{+}$fibers were detected, in tFO-stimulated mice (Fig. $4 \mathrm{e}_{4}, \mathrm{f}_{4}$ ). These results indicate that the information perceived by the Trpa1 ${ }^{+}$neurons in the trigeminal and vagus nerves is transmitted to these specific areas of the brainstem.

Hypotherima induced by the trigeminal and vagal Trpa1 pathways. Trpa1-expressing peripheral neurons project their axons to specific areas in the Sp5 and NTS in the brainstem, wherein $c$-fos expression was induced by $\mathrm{tFO}$ administration (Fig. 4). We thus asked whether artificial activation of these Trpa1 $^{+}$neurons might induce hypothermia. We first examined the effect of $\operatorname{Trpa1}^{+}$neurons projecting to the Sp5. A Credependent retrograde adeno-associated virus encoding hM3Dq (a chemogenetic activator) ${ }^{44}$ fused with mCherry (AAVrg-DIOhM3Dq) was injected bilaterally in the dorsal and ventral Sp5 of Trpa1-Cre and control mice (Fig. 5a). Three weeks after injection, mCherry expression was detected in the TG of Trpa1-Cre mice (Fig. 5b). Activation of Trpa1 ${ }^{+}$neurons projecting to the Sp5 by intraperitoneal (IP) injection of clozapine-N-oxide (CNO) led to increased $c$-fos mRNA expression in the dorsal and ventral Sp5 areas (Fig. 5c). Activation of these neurons by $\mathrm{CNO}$ administration induced hypothermia in Trpa1-Cre mice, whereas such effect was not observed in control animals or by saline administration (Fig. 5d-f). Next, we analyzed the effects of artificial activation of Trpa1 ${ }^{+}$neurons projecting to the NTS. We injected AAVrg-DIO-hM3Dq into the NTS of Trpa1-Cre and control mice (Fig. 6a). Three weeks after infection, we confirmed mCherry expression in the VG (Fig. 6b). Artificial activation of $\operatorname{Trpa1}^{+}$neurons projecting to the NTS by CNO administration induced $c$-fos expression in the NTS (Fig. 6c). CNO administration induced hypothermia in Trpa1-Cre mice infected with AAVrg-DIO-hM3Dq into the NTS (Fig. 6d) but not in control mice (Fig. 6e). These results indicate that artificial activation of Sp5 and NTS, which receive axonal projection from Trpa1 ${ }^{+}$ neurons, induces hypothermia.

Diverse activation profiles of TRPA1. In our model, TRPA1 activation in the TG or VG by tFOs transmits danger information to the Sp5 or NTS to induce multiple physiological effects. It 
a
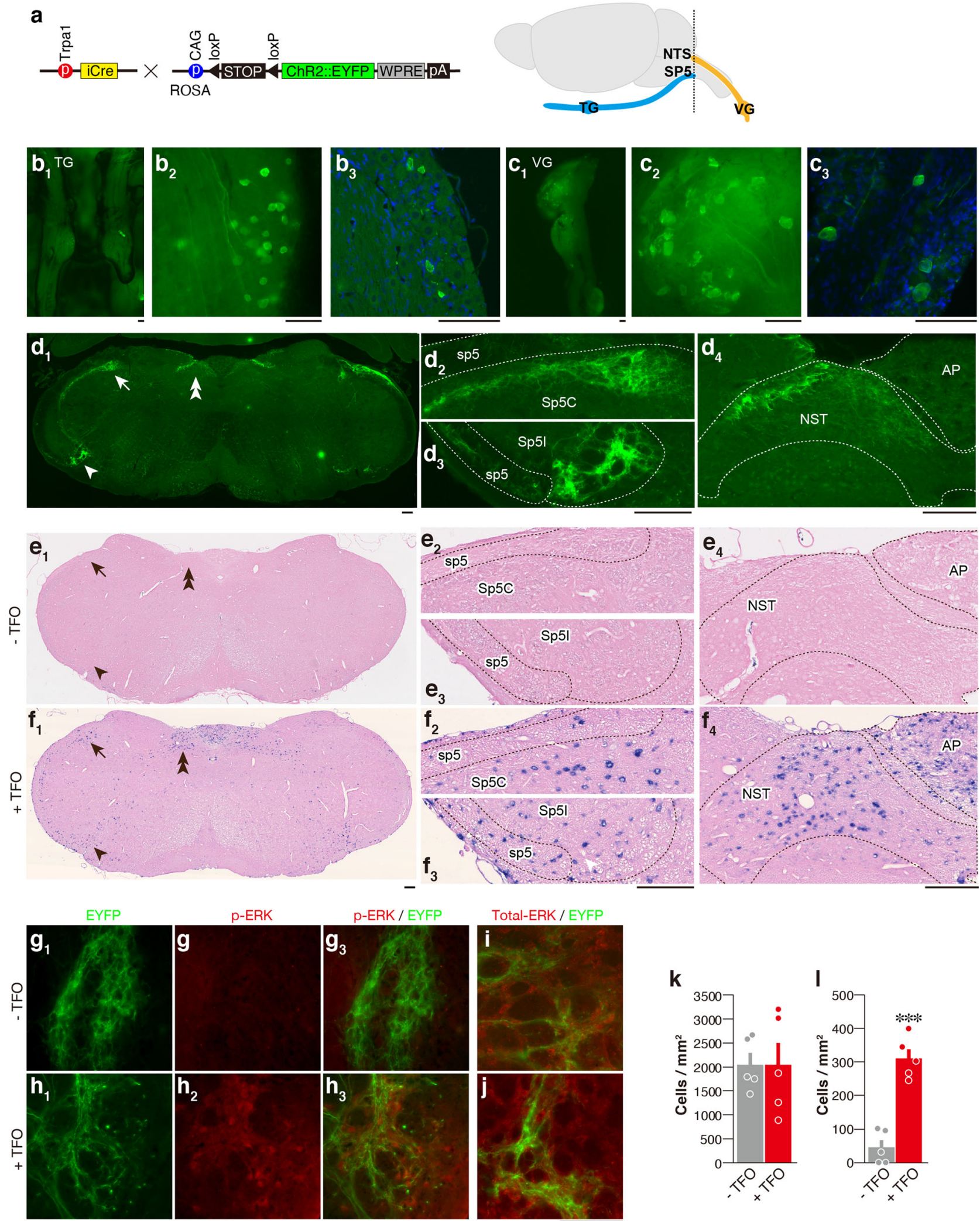

follows that novel compounds inducing more robust effects might be identified by focusing on the molecular activation of TRPA1 and the neuronal activation of TG/VG and Sp5/NTS. We addressed this possibility using known TRPA1 ligands (cinnamaldehyde [CNA] and AITC) and compounds similar to 2MT in chemical structure (4-methyl-2-ethyl-2-thiazoline [4E2MT], 5-methyl-thiazole [5MT], thiomorpholine [TMO], thiophene [TO], and 2-methyl-2-oxazoline [2MO]), for which no antihypoxic activity is known (Fig. 7a).

We first performed inside-out patch-clamp recording to analyze ion currents induced by applying these ligands to TRPA1 expressed in HEK293 cells (Fig. 7b). As reported previously ${ }^{11,45}$, 
Fig. 4 Projection sites of Trpa1 ${ }^{+}$neurons in the Sp5/NTS. a Strategy for selective labeling of Trpa1+ cells using Trpa1-Cre and RCL-ChR2/EYFP mice. Schematic illustration of trigeminal and vagus nerve projections to the brainstem are also shown. A dotted line indicates the approximate position of the sections shown in $\mathbf{d}-\mathbf{j}$. $\mathbf{b}-\mathbf{d}$ Representative EYFP signals in the TG $\left(\mathbf{b}_{\mathbf{1}}-\mathbf{b}_{\mathbf{3}}\right), \mathrm{VG}\left(\mathbf{c}_{\mathbf{1}}-\mathbf{c}_{\mathbf{3}}\right)$, and medulla $\left(\mathbf{d}_{\mathbf{1}}\right)$ of whole mount views $\left(\mathbf{b}_{\mathbf{1}}, \mathbf{c}_{\mathbf{1}}\right)$, magnified wholemount views $\left(\mathbf{b}_{\mathbf{2}}, \mathbf{c}_{\mathbf{2}}\right)$, and tissue sections $\left(\mathbf{b}_{\mathbf{3}}, \mathbf{c}_{\mathbf{3}}, \mathbf{d}_{\mathbf{1}}\right)$ of Trpa1-Cre; RCL-ChR2/EYFP double transgenic mice. Enlarged images of Sp5C ( $\mathbf{d}_{\mathbf{2}} ;$ area indicated by arrow in $\left.\mathbf{d}_{\mathbf{1}}\right)$, Sp5I ( $\mathbf{d}_{\mathbf{3}}$; area indicated by arrowhead in $\left.\mathbf{d}_{\mathbf{1}}\right)$, and NTS ( $\mathbf{d}_{\mathbf{4}}$; area indicated by double arrow in $\left.\mathbf{d}_{\mathbf{1}}\right)$ are also shown. In the Sp5I/C transition area, YFP-positive fibers were observed in the dorsal (arrow) area in the Sp5C and ventral area in the Sp5I (arrowhead) regions ( $\mathbf{d}_{\mathbf{1}}$ ). $\mathbf{e}_{\mathbf{1}}-\mathbf{f}_{\mathbf{4}}$ Representative images of in situ hybridization of c-fos RNA in the medulla following IP injection of saline $\left(\mathbf{e}_{\mathbf{1}}-\mathbf{e}_{\mathbf{4}}\right)$ and tFO (4E2MT; $\mathbf{f}_{\mathbf{1}}-\mathbf{f}_{\mathbf{4}}$ ), along with enlarged images of the Sp5 $\left(\mathbf{e}_{\mathbf{2}}, \mathbf{e}_{\mathbf{3}}, \mathbf{f}_{\mathbf{2}}\right.$, and $\left.\mathbf{f}_{\mathbf{3}}\right)$ and NTS $\left(\mathbf{e}_{\mathbf{4}}, \mathbf{f}_{\mathbf{4}}\right)$. $\mathbf{g}-\mathbf{I}$ Expression of phospho-ERK and total ERK was compared with EYFP signals in the ventral EYFP-fiber-rich area in the Sp5 after IP injection of saline $(\mathbf{g}, \mathbf{i})$ and tFO (4E2MT; $\mathbf{h}, \mathbf{j})$. Quantification of total ERK $(\mathbf{k} ; n=5$ for each, $p=0.9984)$ and $p E R K(\mathbf{I} ; n=5$ for each, $p<0.0001)$ are also shown. Data are shown as mean \pm SEM. Unpaired, two-tailed Student's $t$ test was used to assess significance. Scale bars, $100 \mu \mathrm{m} ;{ }^{\star \star \star} p<0.001$.
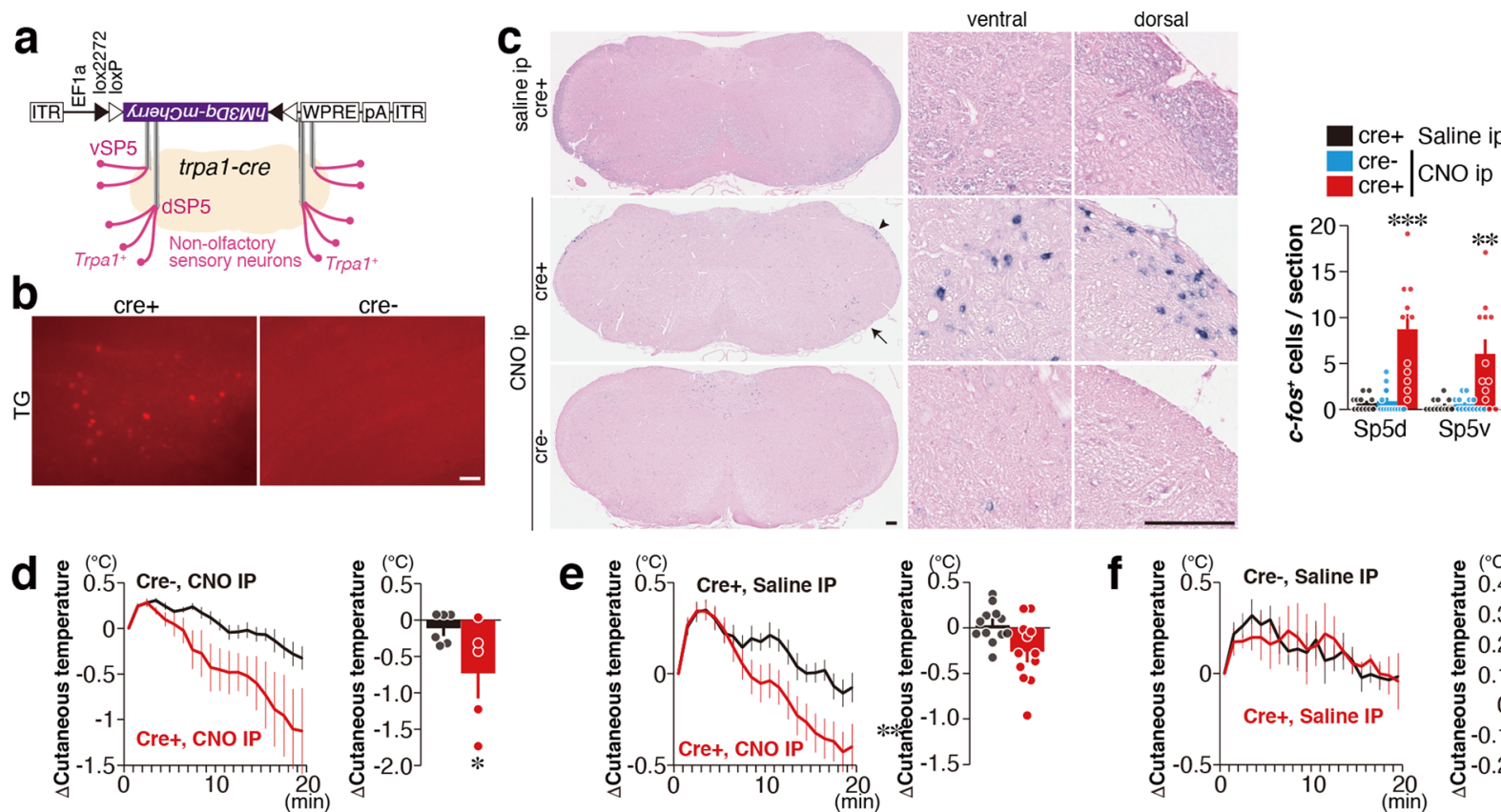

Fig. 5 Hypothermia induced by the Trpa1 ${ }^{+}$neurons projecting to the Sp5. a, b The experimental design of the chemogenetic activation of Trpa1 ${ }^{+}$neurons projecting to the Sp5 (a) and representative retrograde-labeled mCherry expression in the TG in Trpa1-Cre and control mice (b). c Representative images of medulla (left), magnified views in the ventral and dorsal parts of Sp5 (middle; areas indicated by arrow and arrowhead in the left figure), and quantification (right) of $c$-fos ${ }^{+}$cells in the dorsal Sp5 (Sp5d) and ventral Sp5 (Sp5v) after clozapine-N-oxide (CNO) administration (Sp5d, $n=12$ for Trpa1-Cre ${ }^{+} / \mathrm{sal}_{1}, n=$ 14 for Trpa1-Cre $-/ \mathrm{CNO}$, and $n=24$ for Trpa1-Cre ${ }^{+} / \mathrm{CNO}, p=0.9850$ between Trpa1-Cre $+/$ saline and Trp1-Cre $-/ \mathrm{CNO}$, and $p<0.0001$ between Trpa1-Cre + /saline and Trpa1-Cre $+/ \mathrm{CNO}$; Sp5v, $n=12$ for Trpa1-Cre $+/ \mathrm{sal}_{1} n=13$ for Trpa1-Cre $-/ \mathrm{CNO}$, and $n=24$ for Trpa1-Cre $+/ \mathrm{CNO}, p=0.9947$ between $\operatorname{Trpa1-Cre}^{+} / \mathrm{sal}$ and Trpa1-Cre $/ \mathrm{CNO}$, and $p=0.0043$ between Trpa1-Cre $/$ saline and Trpa1-Cre $\left.{ }^{+} / \mathrm{CNO}\right)$ are shown. $\mathbf{d}$ Temporal and mean cutaneous temperature after CNO administration are shown for hM3Dq-infected Trpa1-Cre (red) and control (black) mice ( $n=6$ for control and $n=5$ for Trpa1-Cre, $p=0.0179$ ). e Temporal and mean cutaneous temperature after administration of saline (black) and CNO (red) are shown for hM3Dq-infected Trpa1-Cre mice $(n=12$ each, $p=0.0018)$. f Temporal and mean cutaneous temperature after administration of saline are shown for Trpa1-Cre (red) and control (black) mice ( $n=6$ for control and $n=4$ for Trpa1-Cre, $p=0.500$ ). Data are shown as mean \pm SEM. One-way ANOVA followed by Dunnett's multiple comparison test (c), unpaired one-tailed Student's $t$ test (d), paired one-tailed Student's $t$ test (e), and Mann-Whitney $U$ test (f) were used to assess significance; ${ }^{\star} p<0.05 ;{ }^{\star \star} p<0.01 ;{ }^{\star \star \star} p<0.001$. Scale bars, $100 \mu \mathrm{m}$.

HEK293 cells overexpressing TRPA1 demonstrated channel activities in response to CNA and AITC. Likewise, channel activation was also observed in TRPA1-expressing HEK293 cells in response to 2MT. Among the 2MT-like compounds, 4E2MT, $\mathrm{TMO}$, and $5 \mathrm{MT}$, but not $2 \mathrm{MO}$ or TO, induced channel activation in these cells.

Next, we performed calcium imaging to analyze the ligand response of Trpa1 ${ }^{+}$TG and VG cells by crossing Trpa1-Cre mice with a Cre-dependent GCaMP6-expressing $\operatorname{strain}^{46}$ (Fig. 7c, d). As in the inside-out patch-clamp analyses, $2 \mathrm{MO}$ and TO did not increase calcium influx in Trpa1 ${ }^{+}$TG and VG cells. In contrast, 1 mM CNA and AITC and $10 \mathrm{mM}$ TMO increased calcium influx in both TG and VG Trpa1 ${ }^{+}$cells. While $5 \mathrm{MT}$ had the most robust channel activation response in the inside-out patch-clamp analyses, it did not increase calcium influx in Trpa1 ${ }^{+}$TG and VG cells. 4E2MT led to increased calcium influx in Trpa1 ${ }^{+}$cells only in the VG, whereas 2MT increased calcium influx in those in the TG (Fig. 7c, d). CNA, AITC, and TMO increased calcium influx in a large number of Trpa1 $1^{+}$cells, whereas 4E2MT induced a response in only a small number of $\operatorname{Trpa1}^{+}$cells. These results raise the possibility that Trpa1 ${ }^{+}$cells in the VG and TG may have distinct calcium influx profiles to discriminate closely related molecules.

Then, we measured $c$-fos mRNA in the Sp5 and NTS in response to these ligands (Fig. $8 \mathrm{a}, \mathrm{b}$ ). Both vaporized presentation and IP injection of volatile compounds are known to activate 


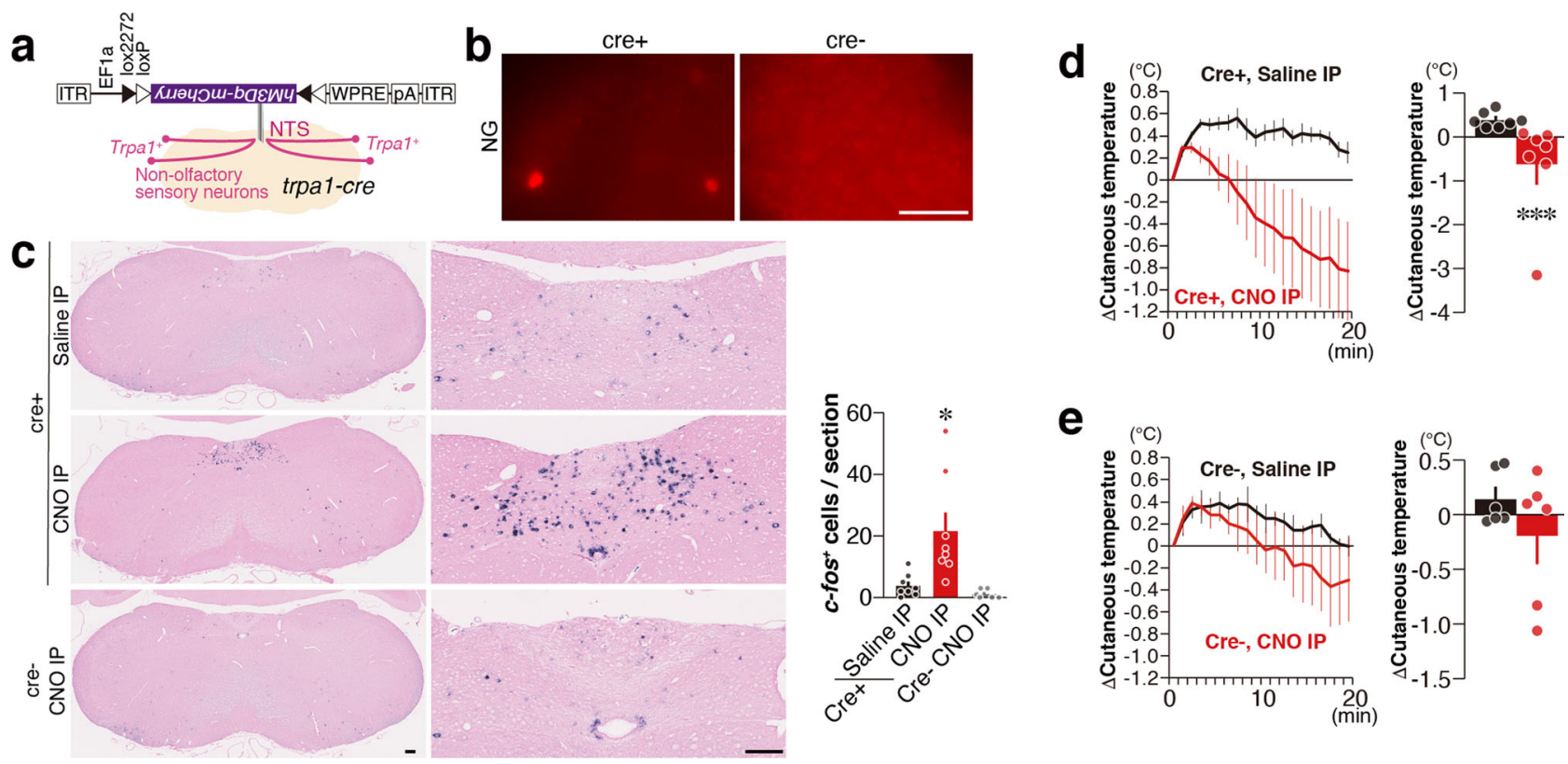

Fig. 6 Hypothermia induced by the Trpa1 ${ }^{+}$neurons projecting to the NTS. a, $\mathbf{b}$ The experimental design of the chemogenetic activation of Trpa $^{+}{ }^{+}$neurons projecting to the NTS (a) and representative retrograde-labeled mCherry expression in the VG in Trpa1-Cre and control mice (b). c Representative images of medulla (left), magnified views in the NTS (middle), and quantification (right) of $c$-fos ${ }^{+}$cells in the NTS after CNO administration are shown ( $n=8$ for Trpa1-Cre ${ }^{+} / \mathrm{sal}$ and Trpa1-Cre ${ }^{+} / \mathrm{CNO}$ and $n=6$ for Trpa1-Cre ${ }^{-} / \mathrm{CNO}, p=0.0135$ between Trpa1-Cre ${ }^{+} /$sal and Trpa1-Cre $^{+} / \mathrm{CNO}$, and $p=0.5335$ between Trpa1-Cre + sal and Trpa1-Cre $/ \mathrm{CNO}$ ). d, e Temporal and mean cutaneous temperature after administration of saline (black) and CNO (red) are shown for hM3Dq-infected Trpa1-Cre (d; $n=7$ each, $p=0.0003)$ and control mice $(\mathbf{e} ; n=6$ each, $p=0.1562)$. Data are shown as mean \pm SEM. Kruskal-Wallis with Dunn's multiple test $(\mathbf{c})$, one-tailed, Mann-Whitney $U$ test $(\mathbf{d})$, and one-tailed, Wilcoxon test (e) were used to assess significance; ${ }^{\star} p<0.05$; ${ }^{* \star} p<0.01$; ${ }^{\star \star \star} p<0.001$. Scale bars, $100 \mu \mathrm{m}$.

sensory neurons ${ }^{47,48}$. We also previously showed that presentation of tFOs both by vapor and IP injection upregulated $c$-fos expression in the Sp5/NTS and also induced hypothermia and anti-hypoxic effects 5 . Thus, we presented these ligands by IP administration in these experiments. Consistent with the patch-clamp and calcium imaging analyses, $2 \mathrm{MO}$ and TO administration did not significantly upregulate $c$-fos in the Sp5 or NTS. Interestingly, among TRPA1 ligands, AITC but not CNA upregulated c-fos expression in the Sp5 and NTS. By contrast, all the 2MT-like compounds, other than 2MO and TO, significantly induced $c$-fos expression in the Sp5 and NTS.

Trpa $1^{+}$sensory neurons project to a particular area in the Sp5/ NTS, and 2MT-induced c-fos mRNA expression in this area was not observed in Trpa1 ${ }^{-1-}$ mice ${ }^{5,9}$ (Supplementary Fig. 4f), suggesting that activation of TRPA1 by $2 \mathrm{MT}$ in the TG/VG might directly induce $c$-fos expression in the Sp5/NTS. However, while $2 \mathrm{MT}$ and its structurally related compounds induced $c$-fos expression in the Sp5/NTS, CNA did not, even though the latter activated TRPA1 like the other compounds.

A possible explanation for this discrepancy is that tFOs activate a different population of $\operatorname{Trpa1}^{+}$neurons which are not activated by CNA, and these neurons project to the Sp5/NTS to induce $c$-fos expression. If this model is true, we would find Trpa1 ${ }^{+}$ sensory neurons which are activated by tFOs but not by CNA. Accordingly, we compared the population of $\operatorname{Trpa1}^{+}$neurons responding to CNA and those responding to tFOs. The responses for CNA and 4E2MT were simultaneously analyzed in 49 Trpa $^{+}$ VG cells (Supplementary Fig. 6). However, we found that almost all Trpa1 ${ }^{+}$VG neurons (48/49 neurons) were more strongly activated by CNA than by $4 \mathrm{E} 2 \mathrm{MT}$. The single remaining neuron responded almost equally to CNA and 4E2MT. We did not find any neurons which were activated by $4 \mathrm{E} 2 \mathrm{MT}$ alone. Although we cannot exclude the possibility that a small subpopulation of Trpa1 $^{+}$cells respond to tFOs but not to CNA, our results did not fit the model that $\operatorname{Trpa1}^{+}$sensory neurons dedicated for tFOs induce $c$-fos expression in the Sp5/NTS. Another possibility is that Sp5/NTS $c$-fos expression is induced by non-TG/VG Trpa1 ${ }^{+}$ sensory inputs which are relevant to tFOs stimulation but not to CNA stimulation. In the unilateral TG ablated mice, $c$-fos expression in the Sp5 in the lesioned side in response to 2MT presentation was suppressed compared to that in the contralateral side, suggesting that $c$-fos expression in the Sp5 is induced by ipsilateral TG projection neurons (Supplementary Fig. 7). In calcium imaging, 4E2MT activated only Trpa1 ${ }^{+}$VG, but not Trpa $1^{+}$TG, neurons (Fig. 7c, d). Thus, it is possible that $c-f o s$ expression in the Sp5/NTS may be induced by 4E2MT, depending on the activation of Trpa1 ${ }^{+}$VG neurons. However, 5MT did not increase calcium influx in most Trpa1 ${ }^{+}$TG/VG neurons (Fig. 7c, d), but it induced $c$-fos expression in the Sp5/NTS (Fig. 8). Thus, we speculate that calcium imaging using isolated TG/VG neurons may not reflect the in vivo response of these cells.

Since tFOs and CNA induce different gene expression in the Sp5/NTS, it is possible that those compounds induce different gene expression also in the TG/VG. To examine this possibility, we compared gene expression in the TG among mice treated with 2MT $(n=2)$, 4E2MT $(n=4)$, TO $(n=2)$, CNA $(n=4)$, AITC $(n=2)$, and saline $(n=2)$ by RNA sequencing (RNAseq). Among the 39,638 genes analyzed, only $12(0.03 \%)$ showed significant differences $(q<0.01)$. Next, we analyzed the correlation among the expressions of these 12 genes and 16 stimulation conditions. Compared to the control (saline) condition, gene expression fluctuations were comparatively smaller in AITC and TO conditions, but larger in 2MT, 4E2MT, and CNA conditions (Fig. 9a). Differentially expressed genes were mainly categorized into two groups. The first group was upregulated in response to 
a<smiles>CC1=NCCS1</smiles><smiles>Cc1cncs1</smiles><smiles>CCC1CSC(C)=N1</smiles><smiles>[Te]=[Te]</smiles><smiles>[C]1[C+]=[C+]C=C1</smiles><smiles>C1#CC1</smiles>

b

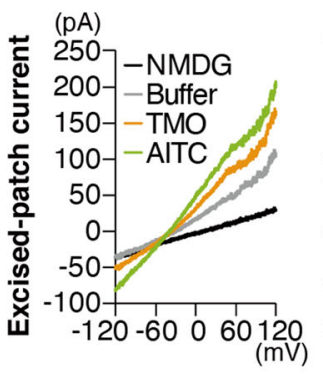<smiles>C1CSCCN1</smiles><smiles>c1ccsc1</smiles><smiles>CC1=NCCO1</smiles><smiles>O[Co]</smiles>
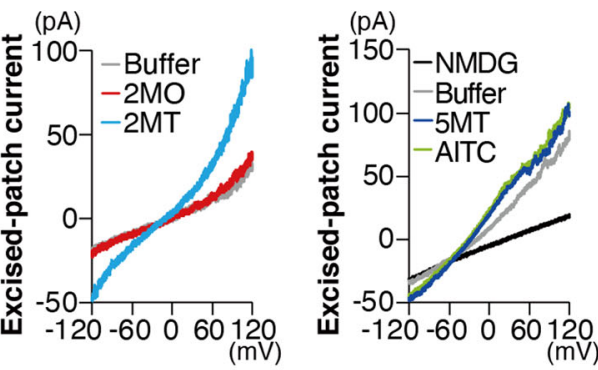

C
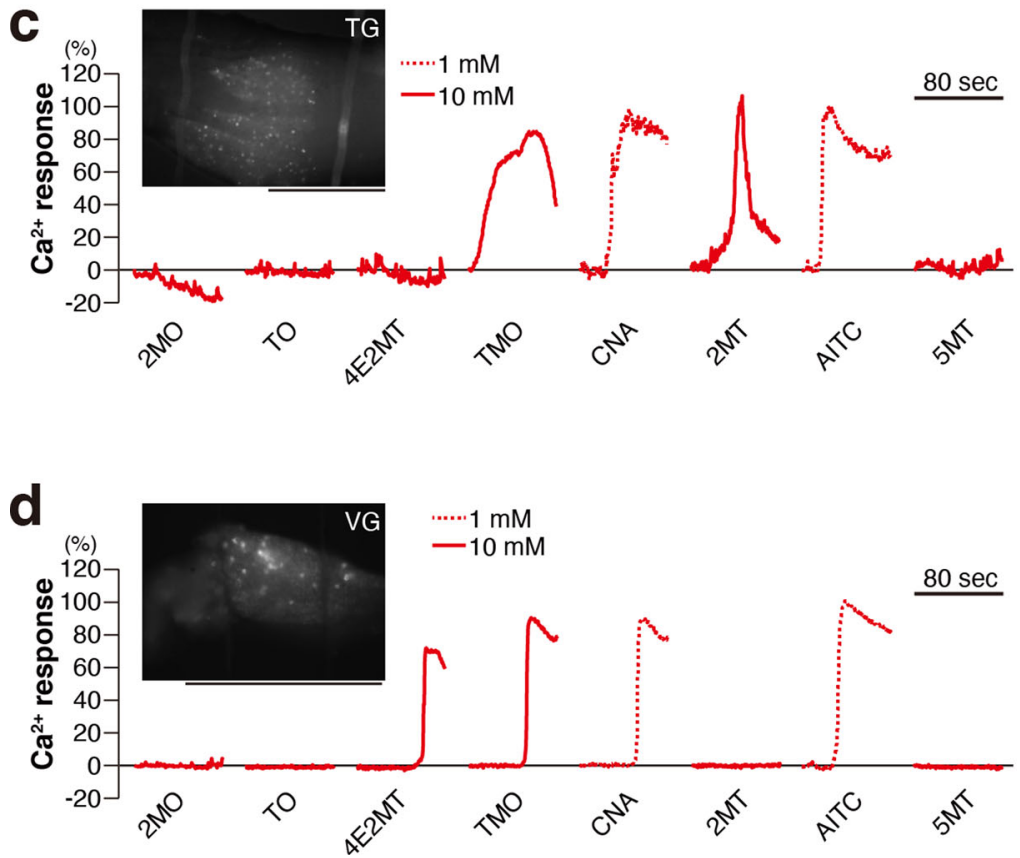<smiles>C=CCN=C=S</smiles>

(\%)
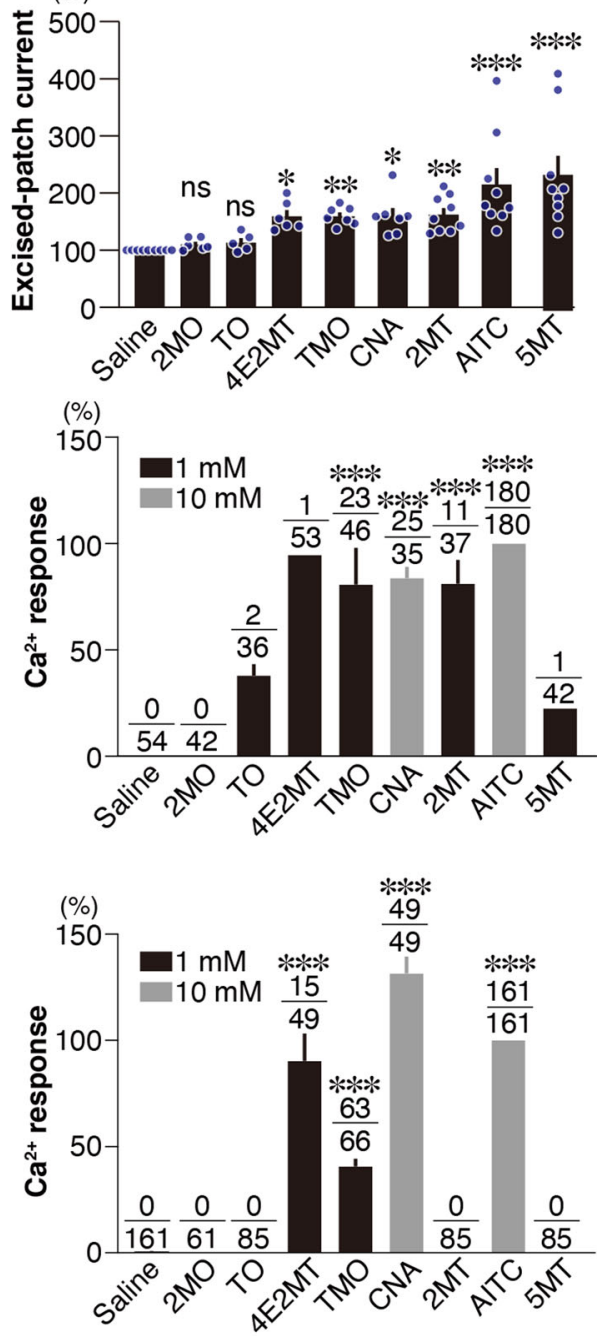

Fig. 7 TRPA1 activation by various compounds. a Chemical structures of tested compounds. b Representative TRPA1 current-voltage (I-V) relations for each compound (left), and relative macroscopic currents from excised patches elicited by application of $100 \mu \mathrm{M}$ of each compound at $+100 \mathrm{mV}$, normalized to those elicited by the application of saline (right; $n=5$ for TO, $n=6$ for $2 \mathrm{MO}$ and $4 \mathrm{E} 2 \mathrm{MT}, n=7$ for TMO and CNA, and $n=9$ for saline, $2 \mathrm{MT}$, AITC, and $5 \mathrm{MT} ; p>0.9999$ for $2 \mathrm{MO}$ and TO, $p=0.018$ for 4E2MT, $p=0.0076$ for TMO, $p=0.0161$ for CNA, $p=0.0041$ for $2 \mathrm{MT}$, and $p<0.0001$ for AITC and 5MT) are shown. c, d (Left) Representative traces of GCaMP6f fluorescence of the TG (c) and VG (d) are shown. A representative image of the TG and VG used in the calcium imaging is also shown. Scale bar, $1 \mathrm{~mm}$. (Right) Calcium activity of AITC-responsive Trpa1 ${ }^{+}$cells in the TG (c) and VG (d) in response to the indicated compounds were analyzed and relative calcium activities of responsive cells for each compound are shown. The number of responded and recorded cells are indicated in the bar graphs. Statistical significance was assessed between activities of all the recorded cells in saline condition and responsive cells for each condition ( $\mathbf{c}, p=0.4916$ for TO, $p=0.3701$ for 4E2MT, $p<0.0001$ for TMO, CNA, 2MT, and AITC, and $p=0.7902$ for 5MT; d, $p<0.0001$ for 4E2MT, TMO, CNA, and AITC). Scale bar, $100 \mu \mathrm{m}$. Data are shown as mean \pm SEM. Kruskal-Wallis with Dunn's multiple comparison test $(\mathbf{b})$ and Kruskal-Wallis with uncorrected Dunn's test $(\mathbf{c}, \mathbf{d})$ were used to assess significance; ${ }^{\star} p<0.05 ;{ }^{\star \star} p<0.01 ;{ }^{\star \star \star} p<0.001$.

2MT and its structurally related compound, 4E2MT (Fig. 9b-e). All five genes categorized into this group were immediate early genes (IEGs). The second group was upregulated in response to CNA and 4E2MT (Fig. 9f-i). Six genes were categorized into this group, and half of them are involved in the regulation of the cytoskeleton and extracellular matrix. These results indicate that different compounds induce differential gene expression in the TG. Both $2 \mathrm{MT}$ and CNA activated Trpa1 ${ }^{+}$cells in the TG. Nevertheless, they induced the expression of distinct sets of genes in the TG. In the current situation, the causal relationship between differential gene expression in the TG and $c$-fos expression in the Sp5/NTS or physiological responses induced by CNA and tFOs are not clarified. It is possible that gene expression in the TG or Sp5/NTS may be useful for prediction of physiological responses of $\mathrm{tFOs.}$

Identification of novel compounds with ultrapotent antihypoxic activities. $2 \mathrm{MO}$ and TO did not activate TRPA1 or 

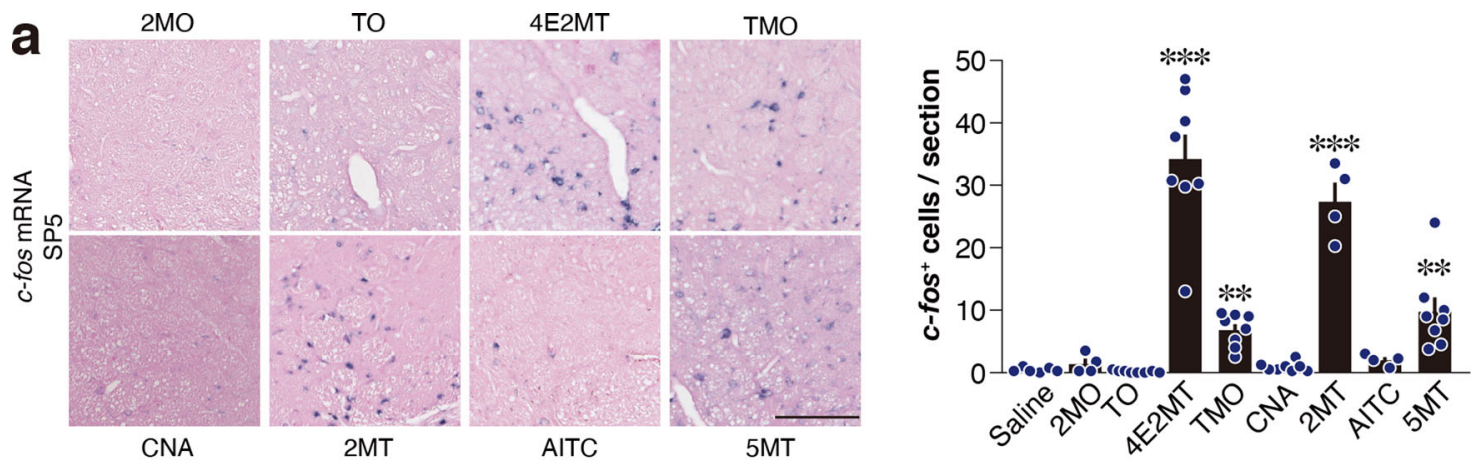

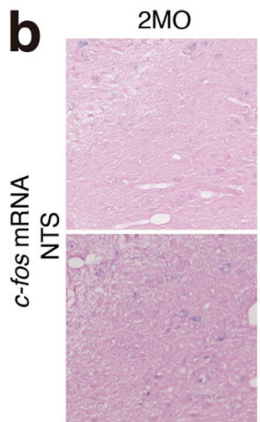

CNA
$\mathrm{TO}$

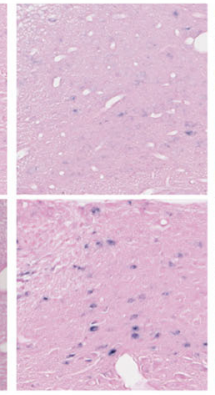

2MT

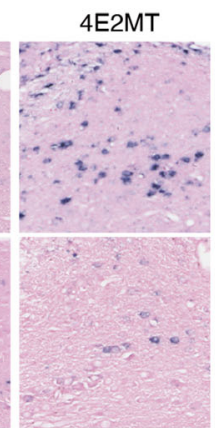

AITC
TMO

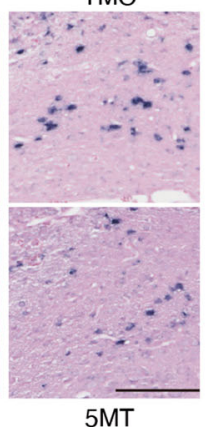

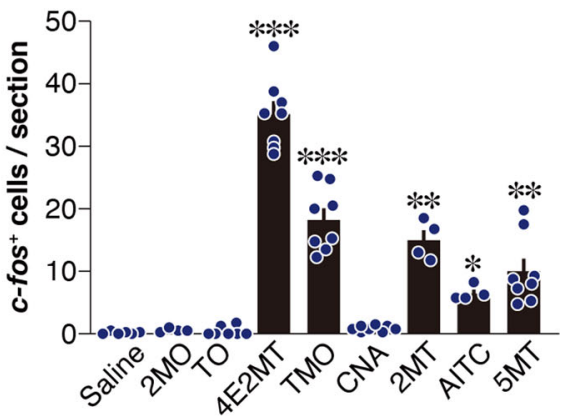

Fig. 8 Sp5/NTS activation by various compounds. a, b Representative images of in situ hybridization of c-fos mRNA (left) and quantification of c-fospositive cells (right) in the Sp5 (a) and NTS (b) in response to IP injection of the indicated compounds ( $n=6$ for saline, $n=4$ for $2 \mathrm{MO}, 2 \mathrm{MT}$, and AITC, and $n=8$ for TO, 4E2MT, TMO, CNA, and 5MT; a, $p=0.5447$ for $2 \mathrm{MO}, p=0.5745$ for TO, $p<0.0001$ for $4 \mathrm{E} 2 \mathrm{MT}, p=0.0071$ for TMO, $p=0.4904$ for CNA, $p=0.0005$ for $2 \mathrm{MT}, p=0.2331$ for AITC, and $p=0.0028$ for $5 \mathrm{MT}$; $\mathbf{b}, p=0.5148$ for $2 \mathrm{MO}, p=0.9379$ for TO, $p<0.0001$ for $4 \mathrm{E} 2 \mathrm{MT}, p=0.0001$ for TMO, $p=0.2798$ for CNA, $p=0.0034$ for $2 \mathrm{MT}, p=0.0447$ for AITC, and $p=0.0043$ for $5 \mathrm{MT}$ ). Scale bar, $100 \mu \mathrm{m}$. Data are shown as mean \pm SEM. Kruskal-Wallis with uncorrected Dunn's test was used to assess significance; ${ }^{\star} p<0.05 ;{ }^{\star \star} p<0.01 ;{ }^{\star \star \star} p<0.001$.

the projection targets of Trpa1 $1^{+}$neurons, Sp5/NTS, in any of the four assays we performed: patch-clamp analysis of TRPA1, calcium imaging of TG/VG, RNAseq analysis of TG, and $c$-fos expression analysis in the Sp5/NTS. By contrast, there were clear differences in the response to known TRPA1 ligands, $2 \mathrm{MT}$, and their structurally related compounds. To determine the assay most predictive of TRPA1-relevant physiological effects, we analyzed the anti-hypoxic effects of these chemicals by IP-injecting them and monitoring survival time in $4 \%$ oxygen for up to $30 \mathrm{~min}$. $2 \mathrm{MO}$ and $\mathrm{TO}$ did not prolong survival time. Importantly, CNA, which activated TRPA1 in the heterologous system and TG/VG cells but did not upregulate IEG genes in TG or $c$-fos in the Sp5/NTS, did not increase survival time, while all other compounds significantly prolonged survival time (Fig. 10a and Supplementary Fig. 8). On the other hand, the present study indicates the involvement of the olfactory system in the regulation of $\mathrm{tFO}$-induced hypothermia. Furthermore, fear-related behaviors to compounds derived from natural predators, e.g., snow leopard urine, in addition to $2 \mathrm{MT}$, TMT, and SBT, are regulated by the olfactory system ${ }^{6,9}$. Thus, it is possible that $c$-fos mRNA expression in the OB could also predict anti-hypoxic activity. To test this possibility, we analyzed the $c$-fos mRNA expression in the OB (Supplementary Fig. 9a-d). 2MT, 4E2MT, and $2 \mathrm{MO}$ greatly increased $c$-fos mRNA expression in the OB. Compared with these compounds, the increase in $c$-fos mRNA expression by TO, CNA, and TMO was weaker. Thus, compared to the increase in c-fos mRNA expression in the TG/VG, the increase in $c$-fos mRNA expression in the $\mathrm{OB}$ was weakly correlated with resistance to hypoxia (Supplementary Fig. 9e). These results indicate that TRPA1 activation in heterologous systems or TG/ VG neurons is insufficient; Sp5/NTS activation is also required to induce anti-hypoxic effects. We further analyzed 4E2MT and TMO as novel ligands showing especially high anti-hypoxic activities.

By prior stimulation with 4E2MT, ATP concentration was maintained in the brain under lethal hypoxic conditions (Supplementary Fig. 10). Whereas 4E2MT stimulation led to robust $\left(>5^{\circ} \mathrm{C}\right)$ hypothermia in $\operatorname{Trpa} 1^{+/-}$mice, this effect was absent in Trpa1 $1^{-/-}$mice (Fig. 10b), as was the prolongation of survival time in $4 \%$ oxygen (Fig. 10c).

TMO was also highly effective in inducing survival in hypoxic conditions: all mice administered TMO survived more than 30 min in $4 \%$ oxygen. To further analyze the extent to which TMO could prolong survival in hypoxic conditions, we measured the length of time each mouse remained alive in hypoxic conditions (Fig. 10d). 2MT stimulation significantly increased survival time in hypoxic conditions compared to control conditions ${ }^{5}$. Compared to $2 \mathrm{MT}$ stimulation, TMO stimulation dramatically prolonged survival time in the $4 \%$ oxygen condition. Accordingly, TMO stimulation suppressed oxygen consumption more prominently than 2MT stimulation (Fig. 8e). Taken together, these results indicate that potent inducers of TRPA1-relevant physiological effects, including hypothermia, hypometabolism, and hypoxia resistance, can be identified by analyzing the activities of TRPA1 and Sp5/NTS (Supplementary Fig. 11).

\section{Discussion}

We focused on Trpa1 as a receptor gene responsible for tFOinduced physiological effects. By a large-scale forward genetic screening, we identified Trpal as a novel target gene inducing freezing behavior in response to 2MT, a type of tFO. Trpa1 regulated not only fear-related behaviors induced by $2 \mathrm{MT}$, but also those induced by a component of fox secretion, TMT, and 

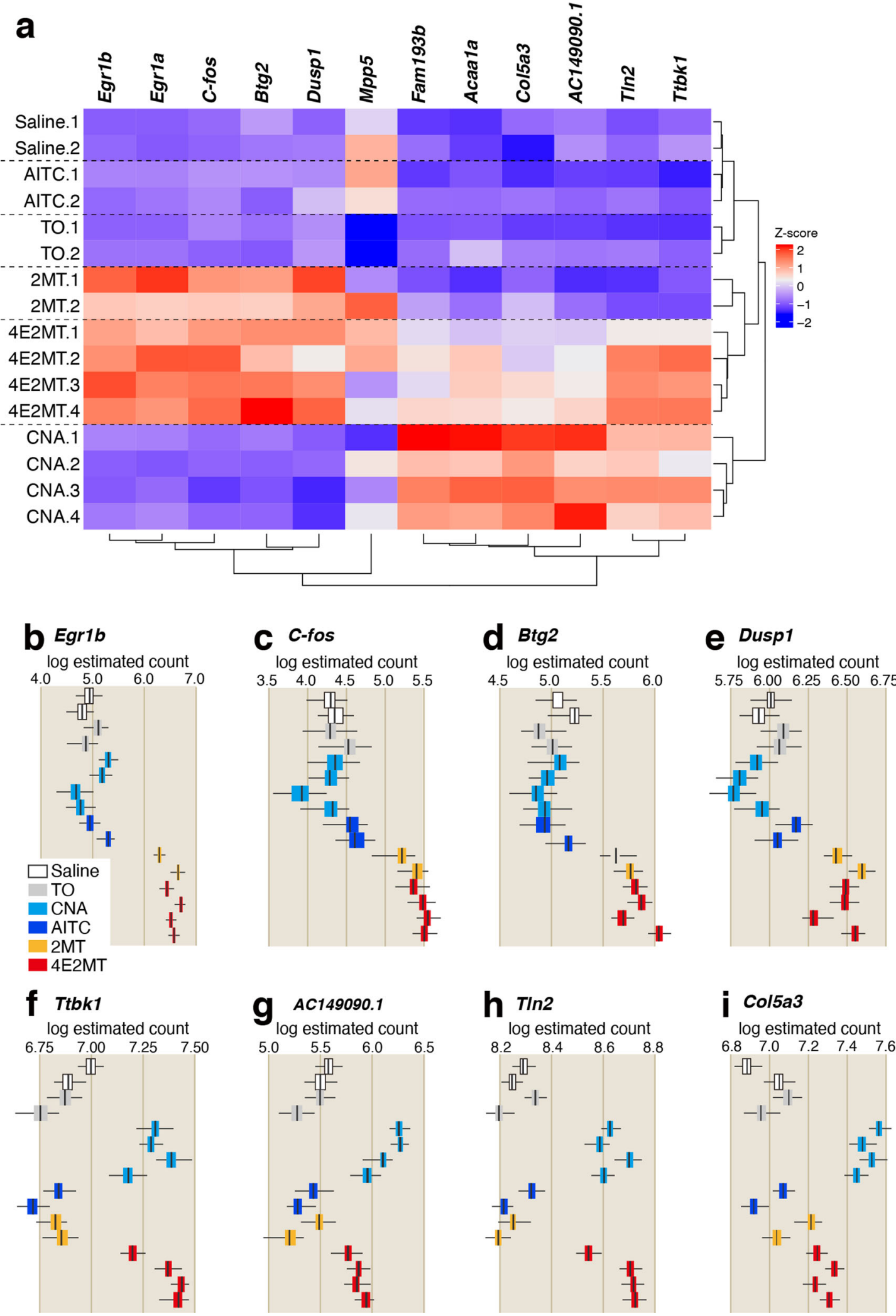

Fig. 9 Compounds induce differential gene expression in the TG. a Clustered heat map depicting relationships across 12 genes, which exhibited significant differences $(q<0.01)$ under 16 stimulation conditions. The color bar indicates the $z$ score scale. Egrla and Egr1b are Ensemble transcript ENSMUST0000006479.5 and ENSMUST00000165033.1, respectively. b-i Box plots depicting the log estimated counts of gene expression in 16 stimulation conditions are shown for the indicated genes that exhibited significant differences $(q<0.01) ; n=2$ for saline, TO, AITC, and $2 \mathrm{MT}$, and $n=4$ for CNA and 4E2M; $q=0.00024$ for Egr1b (b), $q=0.0012$ for $c$-fos (c), $q=0.0024$ for Btg2 (d), $q=0.0067$ for Dusp1 (e), $q=0.0047$ for Tbk1 (f), $q=$ 0.0045 for $A C 149090.1$ ( $\mathbf{g}), q=0.0058$ for $\operatorname{Tln} 2(\mathbf{h})$, and $q=0.0083$ for Col5a3 (i). All boxplots indicate median (center line), 25th and 75th percentiles (bounds of box), and minimum and maximum (whiskers); $q$ value obtained by likelihood ratio test was corrected by Benjamin-Hochberg multiple test. 
a

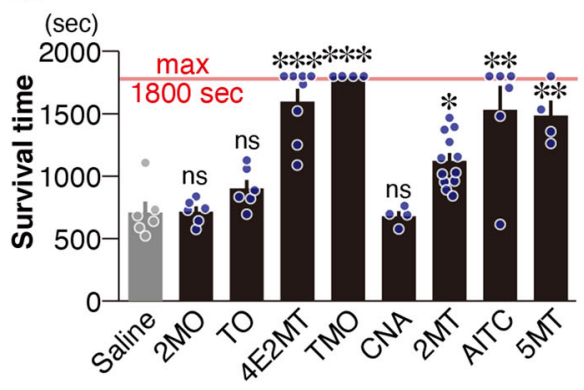

d

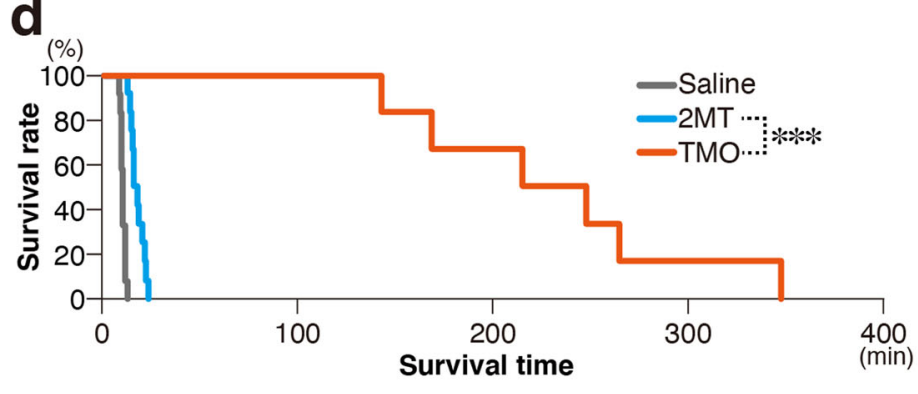

b

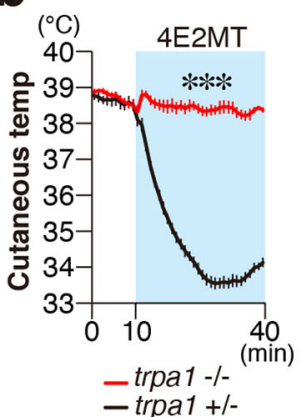

C

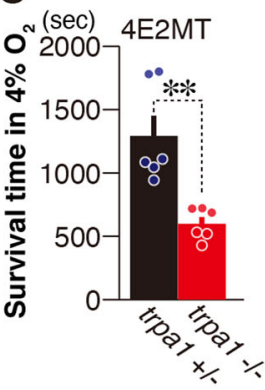

e

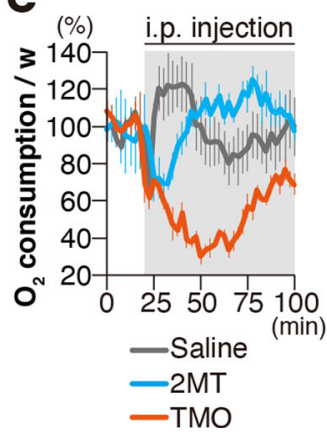

Fig. 10 Identification of novel compounds with ultrapotent anti-hypoxic activities. a Mean survival time in $4 \%$ oxygen in response to IP administration of the indicated compounds are shown $(n=6$ for saline, $2 \mathrm{MT}$, TO, and AITC; $n=8$ for $4 \mathrm{E} 2 \mathrm{MT} ; n=4$ for TMO, CNA, and 5MT; and $n=12$ for $2 \mathrm{MT} ; p=$ 0.8728 for $2 \mathrm{MO}, p=0.2701$ for TO, $p=0.0001$ for $4 \mathrm{E} 2 \mathrm{MT}$ and TMO, $p=0.9493$ for CNA; $p=0.0182$ for $2 \mathrm{MT} ; p=0.0015$ for AITC; and $p=0.0036$ for $5 \mathrm{MT}$, on Kruskal-Wallis with uncorrected Dunn's test). b Temporal analysis of cutaneous temperature in Trpa1-/- (red) and control (black) mice in response to IP administration of 4E2MT are shown ( $n=6$ for each genotype). Statistical significance was assessed for cutaneous temperature after IP administration of 4E2MT (11-40 min; $p<0.0001$, Student's $t$ test, unpaired, one-tailed). c Mean survival time in $4 \%$ oxygen in Trpa $1^{-/-}$(red) and control (black) mice in response to IP administration of 4E2MT ( $n=6$ for each genotype, $p=0.0011$, one-tailed Mann-Whitney $U$ test). $\mathbf{d}$ Survival rate in $4 \%$ oxygen with prior IP administration of saline (gray; $n=6$ ), 2MT (blue; $n=12$ ), and TMO (orange; $n=6$ ). e Temporal analysis of oxygen consumption in response to IP administration of saline (gray), $2 \mathrm{MT}$ (blue), and TMO (orange) ( $n=4$ for each condition). Data are shown as mean \pm SEM; ${ }^{*} p<0.05$; ${ }^{\star \star} p<0.01 ;{ }^{\star \star \star} p<0.001$.

snake skins ${ }^{9}$. Moreover, we demonstrated that Trpa1 is involved in tFO-induced physiological responses including hypothermia, hypometabolism, and hypoxia resistance. We propose a model in which Trpa1 is a central gene in fear perception, responsible for the integrative regulation of behavioral and physiological survivalenhancing responses. On the other hand, tFOs induce fear-related behaviors via the olfactory pathway ${ }^{6,8}$. The present study also indicates that the olfactory pathway is involved in tFO-induced physiological responses. It is possible that in regulating tFOinduced behavioral and physiological responses, there is some separation between the roles of the olfactory pathway mediated by odorant receptors and the trigeminal/vagal pathway mediated by Trpa1. However, their differential roles are currently unknown.

$2 \mathrm{MT}$ stimulation mitigates hypoxic damages and ischemiareperfusion injuries ${ }^{5}$. In the present study, we showed that hypothermia, hypometabolism, and hypoxia resistance induced by $2 \mathrm{MT}$ and other tFOs are partially regulated by Trpal in the trigeminal and vagal nerves. Electrical stimulation of the VNS yields anti-inflammatory effects, and its clinical applications have been proposed. It is considered that VNS yields antiinflammatory effects via artificially intervening inflammatory reflex system, which maintains homeostasis of immune response $^{49}$. Moreover, VNS has therapeutic effects on drugresistant epilepsy and depression. Through its anti-inflammatory responses, it is also expected to have therapeutic effects on a wide spectrum of diseases, including sepsis, cardiovascular disease, traumatic brain injury, stroke, and diabetes ${ }^{50}$. TFOs activate VG neurons and NTS; thus, tFO-stimulation possibly has therapeutic effects comparable to those of VNS. Whether the combination of tFO-TRPA1 has therapeutic effects on various diseases should be examined in a future study.

Different TRPA1-activating ligands induce different physiological responses. For example, AITC, a pungent component of wasabi and mustard oil, induces pain, whereas miR-711 secreted by skin lymphoma induces itch ${ }^{15,51}$. Conversely, acetaminophen and $\Delta^{9}$-tetrahydrocannabinol have antinociceptive effects via Trpal activation ${ }^{25,26}$. Trpal activation is known to be involved in pro-inflammatory responses ${ }^{13,16,45,52}$. On the other hand, Trpa1 agonist CNA was recently reported to have anti-inflammatory effects $^{53}$. These contrasting findings indicate that Trpal induces either positive or negative effects on nociception and inflammatory responses, depending on the ligand stimulus. We demonstrated that CNA induced ion currents in Trpa1-transfected HEK293 cells and calcium influx in $\operatorname{Trpa1}^{+}$TG and VG cells, consistent with previous studies ${ }^{45}$; nevertheless CNA demonstrated low anti-hypoxic effects. For clinical applications, it is important to identify novel ligands capable of inducing TRPA1inducing anti-hypoxic effects. Our data indicate that sole induction of ion currents via TRPA1 is not sufficient to induce antihypoxic effects; the induction of neural activation markers in the Sp5/NTS is also necessary. If this principle can be applied to other organisms, including humans, this strategy can be used to screen novel drugs promoting anti-hypoxic effects in clinical applications. 


\section{Methods}

Mice. Male C57BL/6NCr mice were purchased from Japan SLC, Inc (Shizuoka, Japan). The Trpa1 ${ }^{-1-}$ mice (stock number 006401), Trpv1 ${ }^{-1-}$ mice (stock number 003770), Trpal flox mice (stock number 008649), Omp-Cre mice (stock number 006668), RCL-GCamP6f mice (stock number 028865), and RCL-ChR2(H134R)/ EYFP mice (stock number 024109) were purchased from The Jackson Laboratory (Bar Harbor, ME, USA). Trpal-Cre $e^{+}$mice were generated by inserting improved$\mathrm{Cre}^{54}$ at the start codon of the endogenous Trpal locus using CRISPR/Cas9mediated genome editing in the ES cells ${ }^{55}$. RCL-ChR2(H134R)/EYFP mice were crossed with Trpa1-Cre ${ }^{+}$mice to visualize Trpal-Cre ${ }^{+}$cells. The $\Delta \mathrm{D}$ mice were generated by crossing OMACS-Cre and Eno2-STOP-DTA mice, as reported previously ${ }^{6}$. Advillin-Cre mice were kindly provided by Dr. Wang ${ }^{31}$. Mice were housed under a standard 12-h light/dark cycle at room temperature of $23 \pm 2{ }^{\circ} \mathrm{C}$ with humidity of $30-70 \%$ and allowed ad libitum access to food and water. Mice were at least 9 weeks old at the start of testing. The experimental protocols were approved by the Animal Research Committee of Kansai Medical University.

Chemical compounds. We purchased 2MT, 4E2MT, 5MT, TMO, TO, 2MO, CNA, and AITC from Tokyo Chemical Industry Co., Ltd. (Tokyo, Japan). The vaporized compound presentation was performed by introducing a piece of filter paper containing $271 \mu \mathrm{mol}$ of a test compound into the test cage. Administration of the compound was performed by intraperitoneally injecting $100 \mu \mathrm{l}$ of $1 \%$ solution in saline $(\sim 40 \mathrm{mg} / \mathrm{kg})$. 2MT and TMO can be dissolved in saline at $1 \%$. For the other compounds, because of their low solubility in saline, $1 \%$ of the compound was added to saline and stirred vigorously by vortex to ensure that the compound was dispersed in the saline just prior to IP administration. Presentation and administration of chemical compounds were performed in an open cage placed in a chemical fume hood to avoid cross-contamination.

Measurement of cutaneous temperature. For measurement of cutaneous temperature, mice were anesthetized with pentobarbital $(50 \mathrm{mg} / \mathrm{kg}, \mathrm{IP}) 2-3$ days prior to testing, and the fur on the back was removed with a chemical hair remover. Each mouse was placed in a separate test cage $(17.5 \times 10.5 \times 15 \mathrm{~cm})$ without a lid placed in a chemical fume hood, habituated for $10 \mathrm{~min}$, and subjected to presentations of filter paper containing test compounds (Figs. 1, 2a, b, 3a-f, and Supplementary Figs. 1-4) or IP administration (Figs. 4, 6e, f, 7, 8, and Supplementary Figs. 6-8) of test compounds. Cutaneous temperature was recorded using an infrared digital thermographic camera (H2640; NEC Avio Infrared Technologies Co., Ltd., Tokyo, Japan) and TH92-707 data capture program 1.2(J) (NEC Avio Infrared Technologies Co., Ltd.) at 5 frames/s. Cutaneous temperature on the back was automatically analyzed using specially designed software based on a previously reported method $^{56}$.

Measurement of core body temeperature and heart rates. Measurement of core body temperature and heart rate was performed using a radio-telemetry transmitter (TA11ETA-F10; DataSciences International, St Paul, MN, USA) and Dataquest A.R.T. 4.30 (DataSciences International) according to a method ${ }^{5}$. Briefly, a radio-telemetry transmitter was implanted following the surgical procedure described by the manufacturer. After the surgery, the mice were allowed to recover for $\sim 10$ days before testing. On the test day, each mouse was placed in a separate test cage $(17.5 \times 10.5 \times 15 \mathrm{~cm})$ without a lid placed in a chemical fume hood, habituated for $10 \mathrm{~min}$, and subjected to presentation of filter paper containing test compound (Fig. 1b, e and Supplementary Fig. 1).

For the restrained condition, mice were implanted with radio-telemetry probes approximately 10 days prior to the experiment, as described above. On the test day, each mouse was placed in a separate test cage $(17.5 \times 10.5 \times 15 \mathrm{~cm})$ without a lid, allowed to habituate for $10 \mathrm{~min}$, and physiological parameters were analyzed for 10 min. Immediately afterward, mice were restrained in ventilated $50-\mathrm{ml}$ plastic tubes (Becton Dickinson and Company, Franklin Lakes, NJ, USA), and physiological parameters were analyzed for $30 \mathrm{~min}$ (Fig. 1d, f).

Physiological parameters were automatically transmitted from the device every $10 \mathrm{~s}$ using Dataquest A.R.T. software (DataSciences International).

Measurement of oxygen consumption. Measurement of oxygen consumption was analyzed using a mass spectrometric calorimeter (ARCO-2000, ARCO System, Chiba, Japan) according to a method ${ }^{5}$. Mice were introduced into a metabolic chamber and habituated for more than $60 \mathrm{~min}$. Following habituation, oxygen consumption was measured for $10 \mathrm{~min}$. Afterward, two pieces of filter paper dropped with $25.7 \mu \mathrm{l}$ of $2 \mathrm{MT}$ were presented (Fig. 2a) or $100 \mu \mathrm{l}$ of $1 \%$ test compound $(\sim 40 \mathrm{mg} / \mathrm{kg})$ in saline was IP injected (Fig. 8e), and oxygen consumption was measured.

Hypoxia resistance. Hypoxia resistance was analyzed according to a method ${ }^{5}$. For Fig. $2 \mathrm{~b}$, mice were presented with a filter paper containing $25.7 \mu \mathrm{l}$ of $2 \mathrm{MT}$ in an open cage with a stainless-steel wire bar lid $(26 \times 18 \times 14 \mathrm{~cm})$. Ten minutes after presentation, the mice were moved to a separate test chamber $(17 \times 17 \times 18.5 \mathrm{~cm})$ supplemented with $4 \%$ oxygen. Each chamber had two holes on opposite sides at different heights $(4.5 \mathrm{~cm}$ and $12 \mathrm{~cm}$, respectively) and a wire mesh platform (height: $9 \mathrm{~cm}$ ) where mice were confined for the duration of the experiment. To produce an environment with $4 \% \mathrm{O}_{2}$, compressed nitrogen gas and compressed air cylinders were connected to two gas permeators (PD-1B-2; Gastec Corp., Kanagawa, Japan). A mixture of $1600 \mathrm{ml} / \mathrm{min}$ nitrogen gas and $400 \mathrm{ml} / \mathrm{min}$ air was poured into the test chamber through the upper hole.

For Figs. 2c, d and 8d, mice were intraperitoneally injected with $100 \mu \mathrm{l}$ of $1 \%$ test compound and introduced into separate cages. Thirty minutes after injection, the mice were moved to a separate test chamber $(17 \times 17 \times 18.5 \mathrm{~cm})$ supplemented with $4 \%$ oxygen.

For Supplementary Fig. 6, mice were intraperitoneally injected with $200 \mu \mathrm{l}$ of concentrations $(0.01 \%, 0.1 \%, 1 \%$, and $10 \%)$ of CNA in saline. Thirty minutes after injection, the mice were moved to a separate test chamber $(17 \times 17 \times 18.5 \mathrm{~cm})$ supplemented with $4 \%$ oxygen.

The $\Delta^{9}$-THC was kindly provided by Ruri Hanajiri and acetaminophen was purchased from TCI.

IEG mapping. IEG mapping was conducted by performing in situ hybridization using antisense RNA probes for $c$-fos, according to a method ${ }^{5}$. For Figs. 4e, f, 6e, f, and Supplementary Fig. 7, C57BL/6 mice were introduced into a separate cage $(29 \times 19 \times 13 \mathrm{~cm})$ with a metal with a stainless-steel wire bar lid and habituated for $2 \mathrm{~h}$. Following habituation, mice were intraperitoneally injected with $100 \mu \mathrm{l}$ of saline or $1 \%$ solution of indicated compound in saline $(\sim 40 \mathrm{mg} / \mathrm{kg})$. After $30 \mathrm{~min}$ of IP injection, mice were sacrificed, and coronal brain sections were prepared.

For Fig. 5c, i, AAV-injected animals were introduced into a separate cage $(29 \times$ $19 \times 13 \mathrm{~cm}$ ) with a stainless-steel wire bar lid and habituated for $2 \mathrm{~h}$. Following habituation, mice were intraperitoneally injected with $5 \mathrm{mg} / \mathrm{kg}$ of CNO (SigmaAldrich) or saline. After 30 min of IP injection, mice were sacrificed, and coronal brain sections were prepared.

For Supplementary Fig. 4, Trpa1 ${ }^{-1-}$ and control mice were introduced into a separate cage $(29 \times 19 \times 13 \mathrm{~cm})$ with a metal with a stainless-steel wire bar lid and habituated for $2 \mathrm{~h}$. After habituation, a filter paper dropped with $25.7 \mu \mathrm{l}$ of saline or $2 \mathrm{MT}$ was presented every $5 \mathrm{~min}$ for a 30 -min period. Following $30 \mathrm{~min}$ of presentation, mice were sacrificed, and coronal brain sections were prepared.

For preparing brain sections, mice were anesthetized with gaseous isoflurane (Mylan, Canonsburg, PA) and perfused with ice-cold 4\% paraformaldehyde (PFA) in phosphate-buffered saline (PBS). The brains were then removed and immersed in $4 \%$ PFA in PBS overnight at $4{ }^{\circ} \mathrm{C}$. The fixed brains were dehydrated in a graded ethanol and xylene series and then embedded in paraffin using an automated system (Sakura rotary, RH-12DM; Sakura Finetek, Tokyo, Japan). Coronal sections with a thickness of $5 \mu \mathrm{m}$ were prepared using an automatic slide preparation system (AS-200S, Kurabo, Osaka, Japan).

In situ hybridization was performed using an automated system (Discovery XT, Ventana Medical Systems, Oro Valley, AZ) according to the manufacture's protocol. The digoxigenin (DIG; Roche, Germany) labeled antisense RNA probes for $c$-fos were prepared from the plasmid containing DNA fragments spanning the 129 to 537 and the 543 to 1152 bp regions of mouse $c$-fos according to the manufacture's protocol. The DIG-labeled probes (1:1000 dilution) were hybridized for $3 \mathrm{~h}$ using a RiboMap Kit (Roche) at $74^{\circ} \mathrm{C}$. The slides were then incubated with biotin conjugated anti-DIG antibody (1:500, Jackson ImmunoResearch, West Grove, PA) at $37^{\circ} \mathrm{C}$ for $28 \mathrm{~min}$. The probe was detected using the Ventana BlueMap Kit (Roche, Basel, Switzerland) at $37^{\circ} \mathrm{C}$ for $6 \mathrm{~h}$, and counterstained with a Red counterstain kit (Roche) at $37^{\circ} \mathrm{C}$ for $4 \mathrm{~min}$. Coverslips were applied using an automated system (Tissue Tek ${ }^{\circledast}$ GlasTM; Sakura Finetek) The stained images were scanned using a NanoZoomer virtual microscope system (2.0 RS, NDP.scan 2.5, and NDP. View2, Hamamatsu Photonics, Hamamatsu, Japan). The number of $c$-fos ${ }^{+}$cells in the stained images was then counted by single-blinded investigators.

Histology. For whole-mount analysis of Trpa1-Cre+ cells in TG and VG, EYFP signals from Trpa1-Cre/RCL-ChR2(H134R)/EYFP were observed using a BZ-9000 fluorescence microscope using BZ-II software (Keyence, Osaka, Japan).

For the immunohistochemical analysis of Trpa1-Cre $e^{+}$cells, coronal paraffinized sections were prepared from Trpa1-Cre/RCL-ChR2(H134R)/EYFP mice and deparaffinized with xylene followed by rehydration with a graded ethanol series. After incubation in blocking buffer (5\% goat serum/0.3\% Triton X-100/PBS) for 30 $\mathrm{min}$ at room temperature, the slides were incubated with anti-GFP $(1: 1000, \mathrm{Abcam}$ Cambridge, MS) in blocking buffer for overnight at $4{ }^{\circ} \mathrm{C}$, followed by incubation with anti-rabbit antibody conjugated with Alexa fluor 488 (1:800, Invitrogen, Carlsbad, CA) for $1.5 \mathrm{~h}$.

For the analysis of phospho-ERK (p-ERK), Trpa1-Cre/RCL-ChR2(H134R)/ EYFP or Trpa1-Cre/RCL-GCamP6f mice were habituated for $2 \mathrm{~h}$ in a test cage, then $1 \% 4 \mathrm{E} 2 \mathrm{MT}$ or saline was injected $(100 \mu \mathrm{l}, \mathrm{IP} ; 40 \mathrm{mg} / \mathrm{kg})$. Four minutes after the injections, mice were perfused with $4 \%$ PFA in PBS, and brains were harvested. Brains were post-fixed and immersed in $30 \%$ sucrose/PBS overnight, then embedded in OCT compound; $30-\mu \mathrm{m}$-thick sections were incubated in blocking buffer ( $5 \%$ goat serum/0.3\% Triton X-100/PBS) for $30 \mathrm{~min}$ at room temperature, then incubated with anti-p-ERK (1:200, Cell Signaling Technology, Tokyo, Japan) and anti-GFP (1:1000, nacalai tesque, Kyoto, Japan) in blocking buffer for overnight at $4{ }^{\circ} \mathrm{C}$, followed by incubation with anti-rabbit antibody conjugated with Cy3 (1:800, Jackson ImmunoResearch, West Grove, PA) and anti-rat antibody conjugated with Alexa Fluor 488 (1:800, Jackson ImmunoResearch, West Grove, 
PA, USA) for $1.5 \mathrm{~h}$. Slides were covered with DAPI-containing mounting medium (Vector Laboratories, Burlingame, CA, USA), and fluorescent images were obtained with a DMI6000 B microscope using LAS AF software (Leica, Wetzlar, Germany).

Surgery. Olfactory bulbectomy was performed as described previously. Briefly, mice were anesthetized via an IP injection of pentobarbital $(50 \mathrm{mg} / \mathrm{kg})$ and bilateral OBs were removed by aspiration through a glass pipette ${ }^{57}$. The unilateral lesion of the TG was performed as described previously ${ }^{9}$.

RTX injection. For the injection of RTX into the TG, RTX (100 ng/ $/$ l, Sigma Aldrich Corp, St. Louis, MO, USA) was infused bilaterally into the TG of C57BL/ $6 \mathrm{NCr}$ mice (coordinates: AP, $-0.1 \mathrm{~mm}$; LR, $\pm 1.1 \mathrm{~mm}, \pm 1.5 \mathrm{~mm}$; DV, $-6.5 \mathrm{~mm}$ from the bregma, in total four sites/mouse, $0.5 \mu \mathrm{l} /$ site) using a $10-\mu \mathrm{l}$ Hamilton syringe mounted on an UltraMicroPump (UMP3; World Precision Instruments LLC, Sarasota, FL, USA) and its controller (Micro4, World Precision Instruments LLC). As a control, saline was infused into the same coordinates ( $n=6$ each). After 1-2 weeks of recovery, the hair on the back was removed and cutaneous temperature was measured as described above.

hM3Dq activation of $\mathrm{Trpa}^{+}$cells projecting to the Sp5 and NTS. Mice were anesthetized and placed on a stereotaxic device (Narishige, Tokyo, Japan) with the head bent downward. An incision was made in the skin of the dorsal neck and muscles were dissected to reveal the membrane overlying the dorsal medulla. A retrograde AAV virus carrying a double-floxed inverted $\mathrm{hM} 3 \mathrm{D}(\mathrm{Gq})$ gene (AAVrg-hSyn1-DIO-hM3D(Gq)-mCherry, Addgene) was infused into the Sp5 or NTS using a glass pipette connected to a Nanoject III (Drummond Scientific Co, Broomall, PA, USA). Injections into ths Sp5 were made bilaterally at a rate of $2 \mathrm{nl} / \mathrm{s}$ at the following coordinates: $-0.5 \mathrm{~mm}$ caudal and $\pm 1.6 \mathrm{~mm}$ lateral from the caudal end of the cerebellum at a depth of $0.5 \mathrm{~mm}$ and $1.1 \mathrm{~mm}$ from the surface (in total 4 sites/mouse and $0.5 \mu \mathrm{l} /$ site). Injections into the NTS were made bilaterally at a rate of $1 \mathrm{nl} / \mathrm{s}$ at the following coordinates: $-0.3 \mathrm{~mm}$ caudal and $\pm 0.2 \mathrm{~mm}$ lateral from the caudal end of the cerebellum at a depth of $0.5 \mathrm{~mm}$ from the surface (in total 2 sites/mouse and $0.08 \mu \mathrm{l} / \mathrm{site}$ ). The hair of the back was removed after at least 3 weeks of recovery, and cutaneous temperature was measured 2-3 days later.

On the test day, mice were intraperitoneally administrated with saline or $5 \mathrm{mg} /$ $\mathrm{kg}$ of $\mathrm{CNO}$ (Sigma-Aldrich) after $10 \mathrm{~min}$ of habituation in a test cage $(17.5 \times 10.5 \times$ $15 \mathrm{~cm}$ ) without a lid. Cutaneous temperature was measured for $20 \mathrm{~min}$.

For $c$-fos quantification, mice received a saline or CNO injection $(5 \mathrm{mg} / \mathrm{kg}$, IP) and transferred to a $4 \%$ oxygen chamber $30 \mathrm{~min}$ later. Mice were perfused with $4 \%$ PFA/PBS after they stopped breathing. The brain was paraffinized and $c$-fos in situ hybridization was performed as described above.

Electrophysiology. HEK293 cells (RCB1637) were obtained from the RIKEN BRC Cell bank. Cells were maintained in Dulbecco's modified Eagle's medium (DMEM) supplemented with $10 \%$ FBS and $1 \%$ penicillin-streptomycin at $37^{\circ} \mathrm{C}$ with $5 \%$ $\mathrm{CO}_{2}$. To obtain HEK293 cells stably expressing TRPA1, cells were transfected with pTRPA1-P2A-mCherry, which express mouse TRPA1 together with mCherry ${ }^{9}$, using Lipofectamine 2000 reagent. Twenty-four hours after transfection, the medium was replaced with DMEM containing $400 \mu \mathrm{g} / \mathrm{ml}$ neomycin. After selection, 30 independent colonies were picked, and mCherry-expressing cells were selected for electrophysiological analysis.

Electrophysiological analysis was performed according to a previous study ${ }^{58}$. Briefly, the day before electrophysiological analysis, $\sim 2 \times 10^{5}$ cells were seeded into a 35-mm dish. Stably transfected HEK293 cells were subjected to inside-out patch voltage clamp voltage experiments using an EPC800 USB patch-clamp amplifier (HEKA Instruments Inc., Holliston, MA, USA).

The ramping protocol consisted of a 1200-ms ramp from -120 to $+120 \mathrm{mV}$ from a holding potential of $0 \mathrm{mV}$ applied every $10 \mathrm{~s}$. Inside-out patches were recorded with a $\mathrm{N}$-methyl-D-glucamine (NMDG) solution in the patch pipette: 150 mM NMDG, $150 \mathrm{mM} \mathrm{HCl}, 5 \mathrm{mM}$ EGTA, $0.61 \mathrm{mM} \mathrm{MgCl}_{2}$ to obtain $0.5 \mathrm{mM}$ free $\mathrm{Mg}^{2+}, 0.13 \mathrm{mM} \mathrm{CaCl}_{2}$ to obtain $10 \mathrm{nM}$ free $\mathrm{Ca}^{2+}$ (calculated using maxchelator, http://maxchelator.stanford.edu), and $5 \mathrm{mM}$ HEPES, $\mathrm{pH}$ 7.4. Compounds were dissolved in low $\mathrm{Ca}^{2+}$ solution, that was also used for perfusion of the intracellular side of the patch, containing: $150 \mathrm{mM} \mathrm{NaCl}, 5 \mathrm{mM}$ EGTA, $0.61 \mathrm{mM} \mathrm{MgCl}$ to obtain $0.5 \mathrm{mM}$ free $\mathrm{Mg}^{2+}, 0.13 \mathrm{mM} \mathrm{CaCl}_{2}$ to obtain $10 \mathrm{nM}$ free $\mathrm{Ca}^{2+}$, and $5 \mathrm{mM}$ HEPES, pH 7.4. To confirm the quality of the recordings, we regularly substituted all cations of the intracellular side solution with $\mathrm{NMDG}^{+}$, which is expected to reduce the current to nearly background level ${ }^{59}$. Experiments were performed at a constant temperature $\left(>25^{\circ} \mathrm{C}\right)$.

\section{Calcium imaging of isolated TG and VG}

Animals and imaging session. TrpA1-Cre/RCL-GCamP6f mice (5-12 weeks old) were decapitated, and their TG or VG were isolated and transferred in oxygenated extracellular solution containing (in mM): 20 HEPES, $124 \mathrm{NaCl}, 1.8 \mathrm{KCl}, 1.24$ $\mathrm{KH}_{2} \mathrm{PO}_{4}, 2 \mathrm{MgCl}_{2}, 2 \mathrm{CaCl}_{2}, 10$ D-glucose, $\mathrm{pH}$ 7.4. TG or VG was mounted onto a closed chamber and perfused with the extracellular solution or test solution at a rate of $2 \mathrm{ml} / \mathrm{min}$. TG or VG was first stimulated with $1 \mathrm{mM}$ AITC and then with other agonists sequentially. Each stimulation lasted $25 \mathrm{~s}$, and the intervals were at least $150 \mathrm{~s}$. Imaging was performed with an epifluorescence microscope (MVX10, Olympus, Tokyo, Japan). GCaMP6f-expressing neurons were excited with $460-480 \mathrm{~nm}$ light from a mercury arc lamp (U-LH100HG, Olympus), and the fluorescence was imaged through a $495-540 \mathrm{~nm}$ band-pass filter. Images were acquired with a CMOS camera (acA2040-55um; Basler AG, Ahrensburg, Germany) and Pylon5 software (Basler AG) at $2 \mathrm{~Hz}$.

Data analysis. Fluorescence data were analyzed using ImageJ 1.52 and Excel 2010. Movies were spatially down-sampled by a factor of 4 . Regions of interest (ROIs) of AITC-activated cells were manually drawn around the cell bodies. Artificial trends mainly caused by photobleaching were compensated by fitting the baseline recording period with single exponential curve. Calcium activity was calculated by subtracting the baseline activity (mean fluorescence during $15 \mathrm{~s}$ immediately before application of the agonists) from evoked activity (mean fluorescence during $5 \mathrm{~s}$ before and after the peak). Response to AITC (1 mM) was used as a positive control for $\operatorname{Trpa1}^{+}$cells. For each test compound, responsive cells were defined as cells showing at least one peak higher than the level of negative control (saline).

RNA sequencing. C57BL/6 mice were habituated for $2 \mathrm{~h}$ in a test cage, then intraperitoneally injected with $100 \mu \mathrm{l}$ of $1 \%$ of the test compound in saline ( 40 $\mathrm{mg} / \mathrm{kg}$ ). Following $30 \mathrm{~min}$ of IP injection of the test compound, trigeminal ganglia were dissected, and RNA was extracted using RNeasy Mini Kit (Qiagen) according to the manufacturer's protocol. Library construction and sequencing were performed by Macrogen (Kyoto, Japan). Raw reads were trimmed with Trimmomatic v0.36 (ref. ${ }^{60}$ ) with 'LEADING:30 TRAILING:30 SLIDINGWINDOW:4:15 MINLEN:60' options to keep high-quality sequences, and transcript abundances were quantified by Kallisto v0.44.0 (ref. ${ }^{61}$ ) with 100 bootstraps using the reference transcripts on mouse genome GRCm38 (Ensembl release 93). Differentially expressed transcripts were identified using likelihood ratio test of Sleuth $\mathrm{R}$ package v0.30.0 (ref. ${ }^{62}$ ) with $q$-value of $<0.01$.

Statistics and reproducibility. GraphPad Prism 8 and Microsoft Excel for Mac (version 16.44) were used for statistical analysis. The statistical methods used for each experiment are listed below. The significance level for all the tests was set at $p<0.05$

Figure 1a; D’Agostino-Pearson omnibus test, followed by two-way ANOVA with Sidak's multiple comparison test, was used to assess between $\operatorname{Trpa1} 1^{-1-}$ and $\operatorname{Trpa1}^{+/-}$for cutaneous temperature before and during 2MT presentation. Figure 1b; D'Agostino-Pearson omnibus test, followed by two-way ANOVA with Sidak's multiple comparison test, was used to assess between $\operatorname{Trpa1}^{-1-}$ and $\operatorname{Trpa1}^{+/}$for core temperature before and during $2 \mathrm{MT}$ presentation. Figure 1c; D'Agostino-Pearson omnibus test, followed by two-way ANOVA with Sidak's multiple comparison test, was used to assess between $\operatorname{Trp} v 1^{-1-}$ and $\operatorname{Trp} v 1^{+1-}$ for cutaneous temperature before and during $2 \mathrm{MT}$ presentation. Figure $1 \mathrm{~d}$; D'Agostino-Pearson omnibus test, followed by two-way ANOVA with Sidak's multiple comparison test, was used to assess between $\operatorname{Trpa1^{-1-}}$ and $\operatorname{Trpa1^{+1-}}$ for cutaneous temperature before and during restrained condition. Figure 1e; Anderson-Darling, Shapiro-Wilk, and Kolmogorov-Smirnov tests, followed by two-way ANOVA with Sidak's multiple comparison test, were used to assess between $\operatorname{Trpa1}^{-1-}$ and Trpa1 ${ }^{+/-}$for heart rates before and during 2MT presentation. Figure 1f; D’Agostino-Pearson omnibus test, followed by two-way ANOVA with Sidak's multiple comparison test, was used to assess between $\operatorname{Trpa1}^{-1-}$ and $\operatorname{Trpa1} 1^{+/-}$for heart rate before and during $2 \mathrm{MT}$ presentation. Figure $2 \mathrm{~b}$; the log-rank test was used to assess between $\operatorname{Trpa1^{-1-}}$ and $\operatorname{Trpa1^{+/}}$ for survival time in $4 \%$ oxygen. Figure $2 \mathrm{c}$; the log-rank test was used to assess between saline and $\triangle 9$-THC administrations, between saline and AITC administrations, and between saline and APAP administrations for survival time in $4 \%$ oxygen. Figure 2d; the log-rank test was used to assess between prior IP administration of saline and APAP for Trpa1 $1^{-1-}$ and Trpa1 ${ }^{+/-}$, respetcively. Figure 3a; D'AgostinoPearson omnibus test, followed by two-way ANOVA with Sidak's multiple comparison test, was used to assess between sham and $\mathrm{OBx}$ for cutaneous temperature during presentation of EG and 2MT. Figure 3b; D'Agostino-Pearson omnibus test, followed by two-way ANOVA with Sidak's multiple comparison test, was used to assess between $\Delta \mathrm{D}$ and control for cutaneous temperature during presentation of EG and 2MT. Figure 3c; D'Agostino-Pearson omnibus test, followed by two-way ANOVA with Sidak's multiple comparison test, was used to assess between sham-operated and unilateral TGx mice for cutaneous temperature during presentation of EG and 2MT. Figure 3d; D'Agostino-Pearson omnibus test, followed by two-way ANOVA with Sidak's multiple comparison test, was used to assess between omp-cre/Trpa $1^{\text {flox }}$ and control mice for cutaneous temperature during with and without presentation of $2 \mathrm{MT}$. Figure $3 \mathrm{e}$; Shapiro-Wilk and Kolmogorov-Smirnov tests, followed by two-way ANOVA with Sidak's multiple comparison test, were used to assess between $a d v$-cre/Trpal flox and control mice for cutaneous temperature during with and without presentation of $2 \mathrm{MT}$. Figure $3 \mathrm{f}$; D'Agostino-Pearson omnibus test, followed by two-way ANOVA with Sidak's multiple comparison test, was used to assess between mice which received intra-TG injection of saline and RTX for cutaneous temperature during with and without presentation of 2MT. Figure 4 ; we analyzed $n=5$ for Fig. $4 \mathrm{~b}_{1}, \mathrm{~b}_{2}, n=4$ for Fig. $4 \mathrm{~b}_{3}$ $c_{1}, c_{2}, d, n=3$ for Fig. $4 c_{3}, n=4$ each for Fig. 4 e, f, $n=5$ each for Fig. $4 \mathrm{~g}-\mathrm{j}$, and similar results were obtained. Figure 4k; Shapiro-Wilk and Kolmogorov-Smirnov 
tests, followed by unpaired two-tailed Student's $t$ test, were used to assess between with and without 4E2MT presentation for total $\mathrm{ERK}^{+}$cells in the ventral SP5. Figure 41; Shapiro-Wilk and Kolmogorov-Smirnov tests, followed by unpaired two-tailed Student's $t$ test, were used to assess between with and without 4E2MT presentation for phospho-ERK ${ }^{+}$cells in the ventral SP5. Figure 5b; we stained $n=$ 22 for Trpa1-Cre ${ }^{+}$and $n=2$ for Trpa1-Cre ${ }^{-}$, and obtained similar results. Figure 5c; D'Agostino \& Pearson test, followed by one-way ANOVA with Dunnett's multiple comparison test, was used to assess between saline IP in Trpa1$\mathrm{Cre}^{+}$mice and each of two other conditions. Figure 5d; Kolmogorov-Smirnov test, followed by unpaired one-tailed Student's $t$ test, was used to assess between Trpa1$\mathrm{Cre}^{+}$and control mice infected with AAV for cutaneous temperature after CNO administration. We examined whether cutaneous temperature would be decreased by $\mathrm{CNO}$ administration in the Trpal-Cre $e^{+}$mice compared with that in the control mice. We evaluated the cutaneous temperature reduction using one-tailed test. Figure 5e; D'Agostino \& Pearson test, followed by paired one-tailed Student's $t$ test, was used between saline IP and CNO IP for cutaneous temperature in Trpa1-Cre ${ }^{+}$ infected with AAV. We examined whether cutaneous temperature decreased by $\mathrm{CNO}$ administration. We evaluated the cutaneous temperature reduction using one-tailed test. Figure 5f; Shapiro-Wilk and Kolmogorov-Smirnov tests, followed by Mann-Whitney test, were used between Trpal-Cre ${ }^{+}$and control mice infected with AAV for cutaneous temperature after saline administration. Figure $6 \mathrm{~b}$; we stained $n=8$ for Trpal-Cre ${ }^{+}$and $n=6$ for Trpa1-Cre ${ }^{-}$, and obtained similar results. Figure 6c; Shapiro-Wilk and Kolmogorov-Smirnov tests, followed by Kruskal-Wallis with Dunn's multiple test, were used to assess between $c$-fos ${ }^{+}$cell numbers for saline IP in Trpal-Cre $e^{+}$and each of two other conditions.

Figure 6d; Shapiro-Wilk and Kolmogorov-Smirnov tests, followed by one-tailed Mann-Whitney test, were used between saline and CNO administrations for cutaneous temperature in Trpal-Cre ${ }^{+}$mice infected with AAV. We examined whether cutaneous temperature decreased by $\mathrm{CNO}$ administration. We evaluated the cutaneous temperature reduction using one-tailed test. Figure 6e; Shapiro-Wilk and Kolmogorov-Smirnov tests, followed by one-tailed Wilcoxon matched pairs signed rank test, were used between saline and CNO administrations for cutaneous temperature in control mice infected with AAV. We examined whether cutaneous temperature decreased by $\mathrm{CNO}$ administration. We evaluated the cutaneous temperature reduction using one-tailed test. Figure $7 \mathrm{~b}$; Kolmogorov-Smirnov tests, followed by Kruskal-Wallis with Dunn's multiple comparisons, were performed between excised-patch currents induced by saline and each of test compounds for excised patch-current. Figure 7c;

Kolmogorov-Smirnov test followed by Kruskal-Wallis with uncorrected Dunn's test was performed between calcium responses for all recorded cells in saline condition and those in responsive cells for each of the test compounds. Figure $7 \mathrm{~d}$; Kolmogorov-Smirnov test followed by Kruskal-Wallis with uncorrected Dunn's test was performed between calcium responses for all recorded cells in saline condition and those in responsive cells for each of the test compounds. Figure 8a; Shapiro-Wilk test, followed by Kruskal-Wallis with uncorrected Dunn's test, was performed between saline and each of the test compounds for $c$-fos expression. Figure 8b; Shapiro-Wilk test, followed by Kruskal-Wallis with uncorrected Dunn's test, was performed between saline and each of the test compounds for $c-f o s$ expression. Figure 9; differentially expressed transcripts were identified using likelihood ratio test of Sleuth R package v0.30.0 (ref. ${ }^{62}$ ) with $q$-value of $<0.01$ Figure 10a; Kolmogorov-Smirnov tests, followed by one-way ANOVA with Dunnett's multiple comparison test, were used to assess between saline and each of the test compounds for survival time. Figure 10b; Shapiro-Wilk and Kolmogorov-Smirnov tests, followed by unpaired one-tailed Student's $t$ test, were used to assess between $\operatorname{Trpa1}^{-/-}$and Trpa1 ${ }^{+/-}$for cutaneous temperature after $4 \mathrm{E} 2 \mathrm{MT}$ administration. IP administration of $4 \mathrm{E} 2 \mathrm{MT}$ led to a reduction in cutaneous temperature. The suppression of this effect was evaluated using onetailed test. Figure 10c; Shapiro-Wilk and Kolmogorov-Smirnov tests, followed by one-tailed Mann-Whitney test, were used to assess between $\operatorname{Trpa1} 1^{-1-}$ and Trpa $^{+/-}$for survival time. IP administration of 4E2MT prolonged survival in $4 \%$ oxygen. The suppression of this effect was evaluated using one-tailed test. Figure 10d; log-rank test was performed between $2 \mathrm{MT}$ and TMO administration for survival rate. Supplementary Fig. 1; Shapiro-Wilk and Kolmogorov-Smirnov tests, followed by unpaired one-tailed Student's $t$ test, were used to assess between sham and $\mathrm{OBx}$ for cutaneous temperature and heart rate in response to 2MT presentation. 2MT presentation led to core temperature reduction. Suppression of this effect was evaluated using one-tailed test. Supplementary Fig. 2; D'Agostino \& Pearson test, followed by unpaired one-tailed Student's $t$ test, was used to assess the difference between the $\Delta \mathrm{D}(\mathrm{cng})$ and control mice. 2MT presentation led to core temperature reduction. Suppression of this effect was evaluated using one-tailed test. Supplementary Fig. 3; Shapiro-Wilk and Kolmogorov-Smirnov tests, followed by Mann-Whitney test, were used to assess between sham and unilateral cervical vagotomy for cutaneous temperature in response to $2 \mathrm{MT}$ presentation;

Shapiro-Wilk and Kolmogorov-Smirnov tests, followed by unpaired one-tailed Student's $t$ test, were used between sham and bilateral ablation of VG below the diaphragm for cutaneous temperature in response to 2MT presentation. 2MT presentation led to core temperature reduction. Suppression of this effect was evaluated using one-tailed test. Supplementary Fig. 4; D’Agostino \& Pearson test, followed by Kruskal-Wallis with Dunn's multiple comparison test, was used to assess between every pairs among three conditions for MnPO, VMPO, and PBN; Shapiro-Wilk and Kolmogorov-Smirnov tests, followed by Kruskal-Wallis with
Dunn's multiple comparison test, were used to assess between every pairs among three conditions for NTS. Supplementary Fig. 5; we analyzed four animals and obtained similar results. Supplementary Fig. 7; we analyzed six sections from three individual animals and obtained similar results. Shapiro-Wilk and

Kolmogorov-Smirnov tests, followed by unpaired one-tailed Student's $t$ test, were used to assess the difference between control and lesion sides. 2MT presentation led to an increase in $c$-fos expression in the Sp5. Suppression of this effect was evaluated using one-tailed test. Supplementary Fig. 8; D’Agostino \& Pearson test, followed by unpaired one-tailed Student's $t$ test, was used to assess between saline IP and $0.01 \%$ CNA IP, Shapiro-Wilk test followed by Mann-Whitney test was used to assess between saline IP and 0.1\% CNA IP; Shapiro-Wilk and Kolmogorov-Smirnov tests, followed by unpaired one-tailed Student's $t$ test, were used to assess between saline IP and 1\% CNA IP, and between saline IP and 10\% CNA IP. We examined whether CNA administration could prolong survival time in $4 \%$ oxygen. This effect was evaluated using one-tailed test. Supplementary Fig. 9d; one-way ANOVA with Dunnett's multiple comparisons test was performed to assess between saline and each compound.

Reporting summary. Further information on research design is available in the Nature Research Reporting Summary linked to this article.

\section{Data availability}

The RNAseq data are available from https://www.hgvd.genome.med.kyoto-u.ac.jp/ repository/MGE0000001.html. The relevant data are available from the corresponding author on reasonable request. Source data are provided with this paper.

Received: 27 May 2020; Accepted: 22 February 2021; Published online: 06 April 2021

\section{References}

1. Golden, F. S., Tipton, M. J. \& Scott, R. C. Immersion, near-drowning and drowning. Br. J. Anaesth. 79, 214-225 (1997).

2. Graham, D. T., Kabler, J. D. \& Lunsford, L. Vasovagal fainting: a diphasic response. Psychosom. Med. 23, 493-507 (1961).

3. Vernet-Maury, E., Polak, E. H. \& Demael, A. Structure-activity relationship of stress-inducing odorants in the rat. J. Chem. Ecol. 10, 1007-1018 (1984).

4. Isosaka, T. et al. Htr2a-expressing cells in the central amygdala control the hierarchy between innate and learned fear. Cell 163, 1153-1164 (2015)

5. Matsuo, $\mathrm{T}$ et al. Artificial hibernation/life-protective state induced by thiazoline-related innate fear odors. Commun. Biol. 4, 101 (2021).

6. Kobayakawa, K. et al. Innate versus learned odour processing in the mouse olfactory bulb. Nature 450, 503-508 (2007).

7. Matsuo, T. et al. Genetic dissection of pheromone processing reveals main olfactory system-mediated social behaviors in mice. Proc. Natl Acad. Sci. USA 112, E311-E320 (2015).

8. Saito, H. et al. Immobility responses are induced by photoactivation of single glomerular species responsive to fox odour TMT. Nat. Commun. 8, 16011 (2017)

9. Wang, Y. et al. Large-scale forward genetics screening identifies Trpal as a chemosensor for predator odor-evoked innate fear behaviors. Nat. Commun. 9, 2041 (2018)

10. Story, G. M. et al. ANKTM1, a TRP-like channel expressed in nociceptive neurons, is activated by cold temperatures. Cell 112, 819-829 (2003).

11. Jordt, S. E. et al. Mustard oils and cannabinoids excite sensory nerve fibres through the TRP channel ANKTM1. Nature 427, 260-265 (2004).

12. McNamara, C. R. et al. TRPA1 mediates formalin-induced pain. Proc. Natl Acad. Sci. USA 104, 13525-13530 (2007).

13. Trevisani, M. et al. 4-Hydroxynonenal, an endogenous aldehyde, causes pain and neurogenic inflammation through activation of the irritant receptor TRPA1. Proc. Natl Acad. Sci. USA 104, 13519-13524 (2007).

14. Andersson, D. A., Gentry, C., Moss, S. \& Bevan, S. Transient receptor potential A1 is a sensory receptor for multiple products of oxidative stress. J. Neurosci. 28, 2485-2494 (2008).

15. Kwan, K. Y. et al. TRPA1 contributes to cold, mechanical, and chemical nociception but is not essential for hair-cell transduction. Neuron 50, 277-289 (2006).

16. Bautista, D. M. et al. TRPA1 mediates the inflammatory actions of environmental irritants and proalgesic agents. Cell 124, 1269-1282 (2006).

17. Eid, S. R. et al. HC-030031, a TRPA1 selective antagonist, attenuates inflammatory- and neuropathy-induced mechanical hypersensitivity. Mol. Pain 4, 48 (2008).

18. Takahashi, N. et al. TRPA1 underlies a sensing mechanism for O2. Nat. Chem Biol. 7, 701-711 (2011). 
19. Pokorski, M., Takeda, K., Sato, Y. \& Okada, Y. The hypoxic ventilatory response and TRPA1 antagonism in conscious mice. Acta Physiol. (Oxf.) 210, 928-938 (2014).

20. Bautista, D. M., Pellegrino, M. \& Tsunozaki, M. TRPA1: a gatekeeper for inflammation. Annu. Rev. Physiol. 75, 181-200 (2013).

21. Nilius, B., Appendino, G. \& Owsianik, G. The transient receptor potential channel TRPA1: from gene to pathophysiology. Pflugers Arch. 464, 425-458 (2012).

22. Blackstone, E., Morrison, M. \& Roth, M. B. H2S induces a suspended animation-like state in mice. Science 308, 518 (2005).

23. Cooper, C. E. \& Brown, G. C. The inhibition of mitochondrial cytochrome oxidase by the gases carbon monoxide, nitric oxide, hydrogen cyanide and hydrogen sulfide: chemical mechanism and physiological significance. J. Bioenerg. Biomembr. 40, 533 (2008).

24. Blackstone, E. \& Roth, M. B. Suspended animation-like state protects mice from lethal hypoxia. Shock 27, 370-372 (2007).

25. Andersson, D. A. et al. TRPA1 mediates spinal antinociception induced by acetaminophen and the cannabinoid $\Delta(9)$-tetrahydrocannabiorcol. Nat. Commun. 2, 551 (2011).

26. Gentry, C., Andersson, D. A. \& Bevan, S. TRPA1 mediates the hypothermic action of acetaminophen. Sci. Rep. 5, 12771 (2015).

27. Brunet, L. J., Gold, G. H. \& Ngai, J. General anosmia caused by a targeted disruption of the mouse olfactory cyclic nucleotide-gated cation channel. Neuron 17, 681-693 (1996).

28. Nagata, K., Duggan, A., Kumar, G. \& García-Añoveros, J. Nociceptor and hair cell transducer properties of TRPA1, a channel for pain and hearing. $J$. Neurosci. 25, 4052-4061 (2005).

29. Nakashimo, Y., Takumida, M., Fukuiri, T., Anniko, M. \& Hirakawa, K. Expression of transient receptor potential channel vanilloid (TRPV) 1-4, melastin (TRPM) 5 and 8, and ankyrin (TRPA1) in the normal and methimazole-treated mouse olfactory epithelium. Acta Otolaryngol. 130, 1278-1286 (2010).

30. Li, J., Ishii, T., Feinstein, P. \& Mombaerts, P. Odorant receptor gene choice is reset by nuclear transfer from mouse olfactory sensory neurons. Nature $\mathbf{4 2 8}$, 393-399 (2004).

31. Hasegawa, H., Abbott, S., Han, B. X., Qi, Y. \& Wang, F. Analyzing somatosensory axon projections with the sensory neuron-specific Advillin gene. J. Neurosci. 27, 14404-14414 (2007).

32. Olah, Z. et al. Ligand-induced dynamic membrane changes and cell deletion conferred by vanilloid receptor 1. J. Biol. Chem. 276, 11021-11030 (2001).

33. Szallasi, A. \& Blumberg, P. M. Vanilloid receptor loss in rat sensory ganglia associated with long term desensitization to resiniferatoxin. Neurosci. Lett. 140, 51-54 (1992).

34. Morrison, S. F. \& Nakamura, K. Central neural pathways for thermoregulation. Front Biosci. (Landmark Ed.) 16, 74-104 (2011).

35. Tan, C. L. et al. Warm-sensitive neurons that control body temperature. Cell 167, 47-59.e15 (2016).

36. Hrvatin, S. et al. Neurons that regulate mouse torpor. Nature 583, 115-121 (2020).

37. Takahashi, T. M. et al. A discrete neuronal circuit induces a hibernation-like state in rodents. Nature 583, 109-114 (2020).

38. Sugimoto, T., Fujiyoshi, Y., Xiao, C., He, Y. F. \& Ichikawa, H. Central projection of calcitonin gene-related peptide (CGRP)- and substance P (SP)immunoreactive trigeminal primary neurons in the rat. J. Comp. Neurol. 378, 425-442 (1997).

39. Sugimoto, T., Fujiyoshi, Y., He, Y. F., Xiao, C. \& Ichikawa, H. Trigeminal primary projection to the rat brain stem sensory trigeminal nuclear complex and surrounding structures revealed by anterograde transport of cholera toxin B subunit-conjugated and Bandeiraea simplicifolia isolectin B4-conjugated horseradish peroxidase. Neurosci. Res. 28, 361-371 (1997).

40. Loewy, A. in Central Regulation of Autonomic Functions (eds Loewy, A. D. \& Spyer, K. M.) 88-103 (Oxford, 1990).

41. King, M. in The Role of the Nucleus of the Solitary Tract in Gustatory (ed Bradley, R. M.) (CRC Press/Taylor \& Francis, 2007).

42. Kim, S. H. et al. Mapping of sensory nerve subsets within the vagal ganglia and the brainstem using reporter mice for Pirt, TRPV1, 5-HT3, and Tac1 expression. eNeuro 7, https://doi.org/10.1523/eneuro.0494-19.2020 (2020).

43. Gao, Y. J. \& Ji, R. R. c-Fos and pERK, which is a better marker for neuronal activation and central sensitization after noxious stimulation and tissue injury? Open Pain. J. 2, 11-17 (2009).

44. Alexander, G. M. et al. Remote control of neuronal activity in transgenic mice expressing evolved G protein-coupled receptors. Neuron 63, 27-39 (2009).

45. Bandell, $M$. et al. Noxious cold ion channel TRPA1 is activated by pungent compounds and bradykinin. Neuron 41, 849-857 (2004)

46. Madisen, L. et al. Transgenic mice for intersectional targeting of neural sensors and effectors with high specificity and performance. Neuron $\mathbf{8 5}$, 942-958 (2015).
47. Nakashima, T., Kidera, K., Miyazaki, J., Kuratomi, Y. \& Inokuchi, A. Smell intensity monitoring using metal oxide semiconductor odor sensors during intravenous olfaction test. Chem. Senses 31, 43-47 (2006).

48. Kikuta, S. et al. Longer latency of sensory response to intravenous odor injection predicts olfactory neural disorder. Sci. Rep. 6, 35361 (2016).

49. Tracey, K. J. Reflex control of immunity. Nat. Rev. Immunol. 9, 418-428 (2009).

50. Johnson, R. L. \& Wilson, C. G. A review of vagus nerve stimulation as a therapeutic intervention. J. Inflamm. Res. 11, 203-213 (2018).

51. Han, Q. et al. miRNA-711 binds and activates TRPA1 extracellularly to evoke acute and chronic pruritus. Neuron 99, 449-463.e446 (2018).

52. Obata, K. et al. TRPA1 induced in sensory neurons contributes to cold hyperalgesia after inflammation and nerve injury. J. Clin. Invest. 115, 2393-2401 (2005)

53. Mendes, S. J. F. et al. Cinnamaldehyde modulates LPS-induced systemic inflammatory response syndrome through TRPA1-dependent and independent mechanisms. Int. Immunopharmacol. 34, 60-70 (2016).

54. Shimshek, D. R. et al. Codon-improved Cre recombinase (iCre) expression in the mouse. Genesis 32, 19-26 (2002).

55. Oji, A. et al. CRISPR/Cas9 mediated genome editing in ES cells and its application for chimeric analysis in mice. Sci. Rep. 6, 31666 (2016).

56. Kutsuna, N. et al. Active learning framework with iterative clustering for bioimage classification. Nat. Commun. 3, 1032 (2012).

57. Slotnick, B. \& Bodyak, N. Odor discrimination and odor quality perception in rats with disruption of connections between the olfactory epithelium and olfactory bulbs. J. Neurosci. 22, 4205-4216 (2002).

58. Macpherson, L. J. et al. Noxious compounds activate TRPA1 ion channels through covalent modification of cysteines. Nature 445, 541-545 (2007).

59. Alpizar, Y. A. et al. Bimodal effects of cinnamaldehyde and camphor on mouse TRPA1. Pflugers Arch. 465, 853-864 (2013).

60. Bolger, A. M., Lohse, M. \& Usadel, B. Trimmomatic: a flexible trimmer for Illumina sequence data. Bioinformatics 30, 2114-2120 (2014).

61. Bray, N. L., Pimentel, H., Melsted, P. \& Pachter, L. Near-optimal probabilistic RNA-seq quantification. Nat. Biotechnol. 34, 525-527 (2016).

62. Pimentel, H., Bray, N. L., Puente, S., Melsted, P. \& Pachter, L. Differential analysis of RNA-seq incorporating quantification uncertainty. Nat. Methods 14, 687-690 (2017).

\section{Acknowledgements}

We are grateful to Fumihiro Eto for providing technical assistance. We are grateful to Drs. Shigetada Nakanishi and Tatsuo Kinashshi for critical comments on the manuscript. This work was supported by the following foundations: JSPS KAKENHI (16K07445 to T.M.; $16 \mathrm{H} 06142$ to T.I.; $20 \mathrm{H} 04849$, $18 \mathrm{H} 02546,17 \mathrm{H} 05586$, and $16 \mathrm{~K} 14558$ to R.K.; 20K20578, 18K19350, 18H04806, and 16H02591, to K.K.); the Japan Science and Technology Agency, A-STEP grant to R.K.; the Takeda Science Foundation (to T.M., T.I. R.K., and K.K.); the Canon Foundation (to K.K.); Mishima Kaiun Memorial Foundation (to T.M.); the Dai-ichi Sankyo Foundation (to R.K. and K.K.); the Naito Foundation (to T.I. and K.K.); the Sumitomo Foundation (to K.K.); the Uehara Foundation (to K.K.); the Asahi Glass Foundation (to K.K.); and the Terumo Foundation (to K.K.).

\section{Author contributions}

K.K. designed the study and experiments. K.K. wrote the manuscript with R.K. T.I. performed the experiments shown in Fig. 1 with L.T., T.M., N.K., and S.M. T.M. performed the experiments represented in Fig. 2 with L.T., R.K., and K.K. T.M performed the experiments shown in Fig. $3 \mathrm{a}-\mathrm{c}$ and Supplementary Fig. 3 with T.I., L.T., A.Y., D.K., R.K., and K.K. T.M. performed the experiment shown in Fig. 3d, e with L.C., C.Y.L., and Q.L. T.M. performed the experiments shown in Fig. 4 with D.K., M.I., R.K., and K.K. T.M performed the experiments depicted in Figs. 5, 6, and Supplementary Fig. S3-S5 with T.I., L.T., A.Y., D.K., R.K., and K.K. A.D. performed the experiments represented in Fig. 7b with M.H. Y.H. performed the experiment shown in Fig. $7 c$, d and Supplementary Fig. 6. T.M. and T.I. performed the experiments presented in Fig. 8a, b with R.K. and K.K. T.M. performed the experiments presented in Fig. 9 with K.H., R.K., and K.K. T.M performed the experiments shown in Fig. 10 with L.T., R.K., and K.K. T.M. performed the experiments presented in Supplementary Fig. 1 with K.K. T.M. performed the experiments presented in Supplementary Fig. 2, with L.T. and R.K. T.I. performed the experiments shown in Supplementary Fig. 4 with A.Y., R.K., and K.K. T.I. performed the experiment shown in Supplementary Fig. 5 with D.K. K.K. performed the experiment shown in Supplementary Fig. 7 with T.M. and R.K. K.K. performed the experiment shown in Supplementary Fig. 8 with R.K. K.K. performed the experiment shown in Supplementary Fig. 9 with T.M., A.Y., and R.K. T.M. performed the experiments presented in Supplementary Fig. 10 with T.M., I.Y., and M.S.

\section{Competing interests}

The authors declare no competing interests. 


\section{Additional information}

Supplementary information The online version contains supplementary material available at https://doi.org/10.1038/s41467-021-22205-0.

Correspondence and requests for materials should be addressed to Q.L., R.K. or K.K.

Peer review information Nature Communications thanks Ioana Carcea, Fernando Martinez-Garcia and the other, anonymous, reviewer(s) for their contribution to the peer review of this work. Peer reviewer reports are available.

Reprints and permission information is available at http://www.nature.com/reprints

Publisher's note Springer Nature remains neutral with regard to jurisdictional claims in published maps and institutional affiliations. (c) (i) Open Access This article is licensed under a Creative Commons Attribution 4.0 International License, which permits use, sharing, adaptation, distribution and reproduction in any medium or format, as long as you give appropriate credit to the original author(s) and the source, provide a link to the Creative Commons license, and indicate if changes were made. The images or other third party material in this article are included in the article's Creative Commons license, unless indicated otherwise in a credit line to the material. If material is not included in the article's Creative Commons license and your intended use is not permitted by statutory regulation or exceeds the permitted use, you will need to obtain permission directly from the copyright holder. To view a copy of this license, visit http://creativecommons.org/ licenses/by/4.0/.

(C) The Author(s) 2021 